Sias et al. 1

\title{
A bidirectional corticoamygdala circuit for the encoding and retrieval of detailed reward memories
}

Ana C. Sias ${ }^{1}$, Ashleigh K. Morse ${ }^{1}$, Sherry Wang ${ }^{1}$, Venuz Y. Greenfield ${ }^{1}$, Caitlin M. Goodpaster ${ }^{1}$, Tyler M. Wrenn ${ }^{1}$, Andrew M. Wikenheiser ${ }^{1-3}$, Sandra M. Holley ${ }^{5}$, Carlos Cepeda ${ }^{5}$, Michael S. Levine ${ }^{2,5}$, Kate M. Wassum ${ }^{1-4}$

1. Dept. of Psychology, UCLA, Los Angeles, CA 90095. 2. Brain Research Institute, UCLA, Los Angeles, CA 90095, USA. 3. Integrative Center for Learning and Memory, University of California, Los Angeles, Los Angeles, CA, USA. 4. Integrative Center for Addictive Disorders, University of California, Los Angeles, Los Angeles, CA, USA. 5. Intellectual and Developmental Disabilities Research Center, Semel Institute for Neuroscience and Human Behavior, Darousevid Geffen Scchool of Medicine, UCLA, Los Angeles, CA 90095, USA.

Correspondence:

Kate Wassum: kwassum@ucla.edu

Dept. of Psychology, UCLA

1285 Pritzker Hall

Box 951563

Los Angeles, CA 90095-1563

Key words: reward, memory, encoding, learning, retrieval, decision making, Pavlovian conditioning, basolateral amygdala, orbitofrontal cortex, Pavlovian-to-instrumental transfer

Figures: 5

Tables: 1 (Key resource table)

Supplemental Tables: 0

Supplemental Figures: 12 


\section{ABSTRACT}

Adaptive reward-related decision making often requires accurate and detailed representation of potential available rewards. Environmental reward-predictive stimuli can facilitate these representations, allowing one to infer which specific rewards might be available and choose accordingly. This process relies on encoded relationships between the cues and the sensory-specific details of the reward they predict. Here we interrogated the function of the basolateral amygdala (BLA) and its interaction with the lateral orbitofrontal cortex (IOFC) in the ability to learn such stimulus-outcome associations and use these memories to guide decision making. Using optical recording and inhibition approaches, Pavlovian cue-reward conditioning, and the outcome-selective Pavlovian-to-instrumental transfer (PIT) test in male rats, we found that the BLA is robustly activated at the time of stimulus-outcome learning and that this activity is necessary for sensory-specific stimulus-outcome memories to be encoded, so they can subsequently influence reward choices. Direct input from the IOFC was found to support the BLA in this function. Based on prior work, activity in BLA projections back to the IOFC was known to support the use of stimulus-outcome memories to influence decision making. By multiplexing optogenetic and chemogenetic inhibition we performed a serial circuit disconnection and found that the IOFC $\rightarrow B L A$ and $B L A \rightarrow I O F C$ pathways form a functional circuit regulating the encoding (IOFC $\rightarrow B L A)$ and subsequent use $(B L A \rightarrow I O F C)$ of the stimulus-dependent, sensory-specific reward memories that are critical for adaptive, appetitive decision making. 
To make good decisions we must accurately anticipate the potential outcomes (e.g., rewarding events) that might be available in our current situation, or state. When not readily observable, we can infer the availability of these outcomes from predictive environmental stimuli (e.g., restaurant logos on a food-delivery app). Pavlovian stimulus-outcome associative memories enable such cues to trigger representations of their associated outcomes, thus facilitating the state-dependent outcome expectations that influence decision making (Balleine \& Dickinson, 1998; Delamater, 2012; Fanselow \& Wassum, 2015). Often our decisions require detailed information about the available outcomes (e.g., flavor, nutritional content, texture). For example, when deciding between items of similar valence (e.g., to have pizza or sushi for dinner). To enable such decisions, stimulusoutcome memories can be quite rich, including the sensory-specific, identifying details of the predicted reward (Delamater \& Oakeshott, 2007; Fanselow \& Wassum, 2015). Failure to properly encode or use such memories can lead to poor reward-related choices, a hallmark feature of myriad psychiatric diseases. Yet much is unknown of the neural circuits that support stimulus-outcome memory.

One potential hub for stimulus-outcome memory is the basolateral amygdala (BLA) (Wassum \& Izquierdo, 2015). Long known for its function in emotional learning, the BLA is thought to link predictive stimuli with valence and to relay that valence for adaptive behavior (e.g., approach/avoidance) (Baxter \& Murray, 2002; Janak \& Tye, 2015; Pignatelli \& Beyeler, 2019; Tye, 2018). But the BLA does more than valence. Mounting evidence, primarily collected with lesion and inactivation strategies, suggests the BLA mediates appetitive behaviors that require a rich sensory-specific representation of the expected reward. For example, the BLA is needed for rewardpredictive cues to bias choice between two distinct rewards (Blundell et al., 2001; Corbit \& Balleine, 2005; Hatfield et al., 1996; Ostlund \& Balleine, 2008). Although the BLA's function in the expression of such behaviors has been established, temporal limitations of BLA lesions preclude interpretations of BLA function in stimulusoutcome learning. The BLA is known to be essential for the learning of cued fear (Muller et al., 1997; Sengupta et al., 2018), but behavioral limitations of these studies preclude understanding of whether the BLA is involved in encoding the sensory-specific details of the outcome. Thus, it remains unknown whether the BLA is involved in encoding the sensory-specific stimulus-outcome memories that enable adaptive choices, or if the BLA primarily functions to assign general valence to a cue. Moreover, little is known of the endogenous activity or circuit function underlying any potential role for the BLA in the formation of appetitive stimulus-outcome memories.

To address these gaps in knowledge, here we used optical recording and inhibition approaches in male rats to examine the BLA's function in the encoding of stimulus-outcome memories for two unique food rewards. To assess the extent of stimulus-outcome memory encoding, we used the outcome-selective Pavlovian-toinstrumental transfer (PIT) test to measure the ability of a reward-paired stimulus to trigger a sensory-specific representation of its predicted reward and thus bias reward-seeking choice (Colwill \& Motzkin, 1994; Corbit \& Balleine, 2016; Gilroy et al., 2014; Kruse et al., 1983).

\section{RESULTS}

\section{BLA neurons respond to rewards and cues during appetitive Pavlovian stimulus-outcome learning.}

We first asked whether and when the BLA is active during the encoding of stimulus-outcome memories (Figure 1a). To condition cues that set the 'state' for a specific reward's availability and engender a sensory-specific representation of that reward, we used a dual food outcome Pavlovian conditioning task. Each of 2, 2-min auditory conditional stimuli (CSs; white noise and tone) were associated with intermittent delivery of 1 of 2 distinct food rewards (sucrose solution or food pellets; e.g., white noise-sucrose/tone-pellet). This conditioning has been shown to engender the encoding of detailed, sensory-specific stimulus-outcome memories as measured by the cue's ability to subsequently promote instrumental choice for the specific predicted reward during a PIT test (Lichtenberg et al., 2017; Lichtenberg \& Wassum, 2016; Malvaez et al., 2015; Ostlund \& Balleine, 2008), as well as the sensitivity of the conditional food-port approach response to sensory-specific devaluation of the predicted 
bioRxiv preprint doi: https://doi.org/10.1101/2021.03.20.436233; this version posted June 9, 2021. The copyright holder for this preprint (which was not certified by peer review) is the author/funder, who has granted bioRxiv a license to display the preprint in perpetuity. It is made available under aCC-BY-NC-ND 4.0 International license.

Sias et al. 4

a

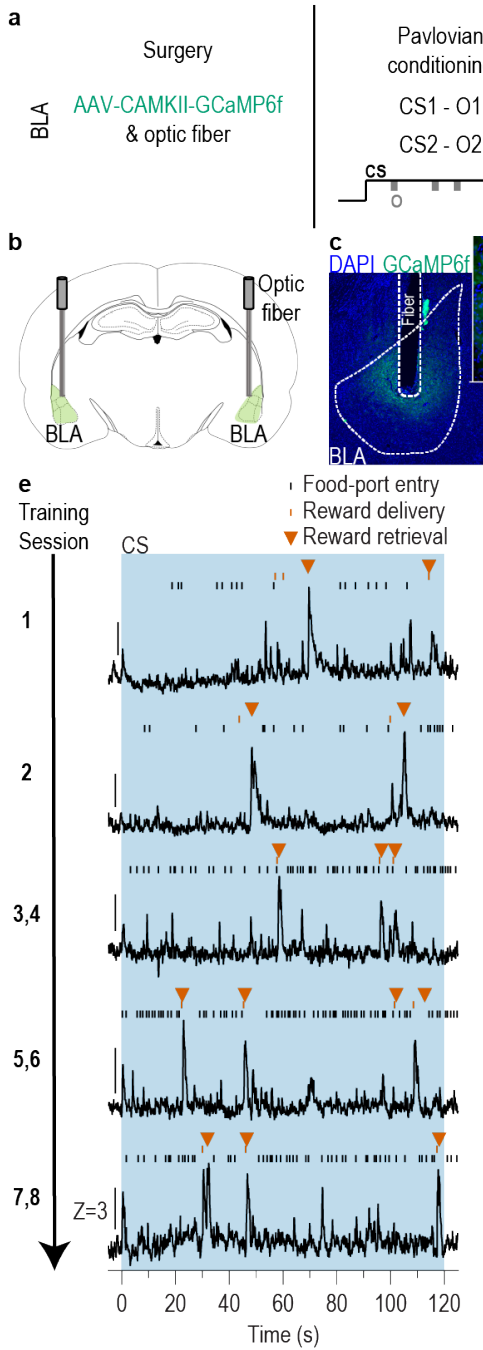

h

i d

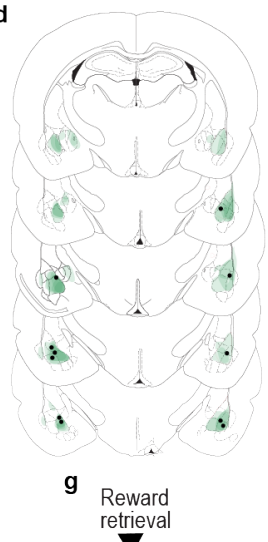

f $\quad \mathrm{CS}$

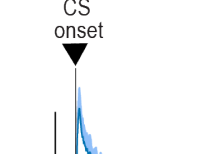

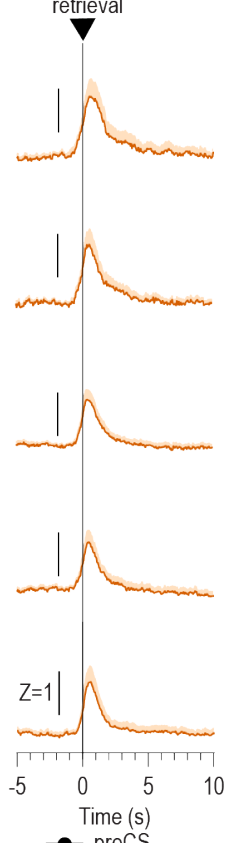

$\rightarrow$ precs

$\rightarrow C S$

- preReward

- Reward retrieval
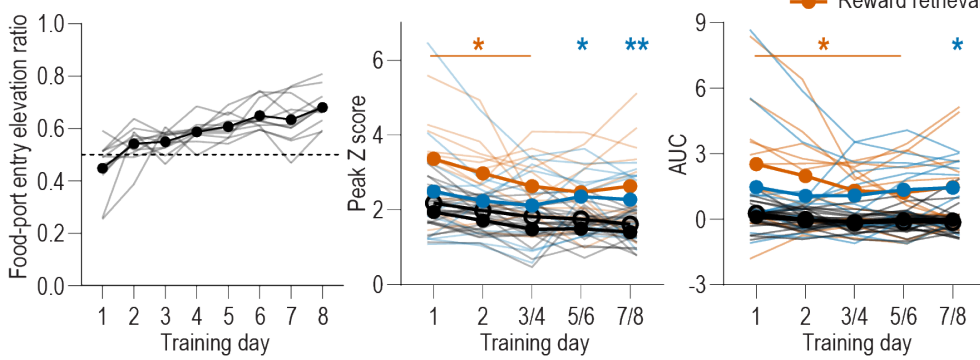

Figure 1. BLA neurons are activated during stimulus-outcome learning. (a) Procedure schematic. CS, conditional stimulus (white noise or tone); $\mathrm{O}$, outcome (sucrose solution or food pellet). (b) Schematic of fiber photometry approach for imaging bulk calcium activity in BLA neurons. (c) Representative fluorescent image of GCaMP6f expression and fiber placement in the BLA. (d) Schematic representation of GCaMP6f expression and placement of optical fiber tips in BLA for all subjects. Brain slides from (Paxinos \& Watson, 1998). (e) Representative examples of GCaMP6f fluorescence changes (Z-scored $\Delta F / F$ ) in response to CS presentation (blue box), reward delivery, and reward retrieval (first food-port entry following reward delivery) across days of training. Traces from the last 6 days of training were selected from 1 of each 2-session bin. See Figure 1-2 for raw GCaMP and isosbestic signal fluctuations. (f-g) Trialaveraged GCaMP6f fluorescence changes (Z-scored $\Delta F / F)$ in response to CS onset (f; blue) or reward retrieval during the CS (g; orange) across days of training. Shading reflects between-subjects s.e.m. Data from the last six sessions were averaged across 2-session bins $(3 / 4,5 / 6$, and $7 / 8)$. (h) Elevation [(CS probe entry rate)/(CS probe entry rate + preCS entry rate)] in food-port entries during the CS probe period (after CS onset, before first reward delivery), averaged across trials and across the 2 CSs for each day of Pavlovian conditioning. Gray lines represent individual subjects. (i-j) Trial-averaged quantification of maximal (i; peak) and area under the GCaMP Z-scored $\Delta F / F$ curve (j; AUC) during the 3-s period following CS onset or reward retrieval compared to equivalent baseline periods immediately prior to each event. Thin light lines represent individual subjects. $N=11$ (see Figure 1-3 for data from $N=8$ subjects with longitudinal data from each session). ${ }^{*} P<0.05,{ }^{* *} P<0.01$ relative to pre-event baseline. 
reward (Lichtenberg et al., 2017) or degradation of the stimulus-outcome contingency (Ostlund \& Balleine, 2008). Food-deprived, male rats $(N=11)$ received 8 Pavlovian conditioning sessions. During each session each cue was presented 4 times (variable intertrial interval, average $=3 \mathrm{~min}$ ) for $2 \mathrm{~min}$, during which its associated reward was intermittently delivered on average every $30 \mathrm{~s}$. Rats demonstrated simple Pavlovian conditioning by gradually increasing their goal approach responses (entries into the food-delivery port) during the cue probe periods (after cue onset, before reward delivery) across training (Figure 1h; Training: $F_{(2.4,24.3)}=13.18, P<$ 0.0001 ; see also Figure 1-1).

To characterize the endogenous activity of BLA neurons during the encoding of appetitive stimulus-outcome memories, we used fiber photometry to image the fluorescent activity of the genetically encoded calcium indicator GCaMP6f (Chen et al., 2013) each day during Pavlovian conditioning (Figure 1b-d). GCaMP6f was expressed preferentially in principal neurons based on expression of calcium/calmodulin-dependent protein kinase, CaMKII (Butler et al., 2011; Tye et al., 2011). Data from the 8 training sessions were binned into 5 conditioning phases, session 1 , session 2 , sessions $3 / 4,5 / 6$, and $7 / 8$. Thus data from the last six sessions were averaged across 2 session bins. As can be seen in the representative examples (Figure 1e; see also Figure 1-2), or group-averaged traces (Figure 1f-g), BLA neurons were robustly activated by both cue onset and reward retrieval (first food-port entry after reward delivery) throughout Pavlovian conditioning. Across training, both the cues and rewards caused a similar elevation in the peak calcium response (Figure 1i; Event v. baseline: $F_{(0.4,3.9)}=36.02, P=0.007$; Training: $F_{(2.8,28.1)}=4.29, P=0.01$; Event type (CS/US) and interactions between factors, lowest $\left.P=0.18\right)$ and area under the calcium curve (AUC; Figure 1j; Event v. baseline: $F_{(0.3,3.4)}=35.23, P=0.01$, Training, Event type, and interactions between factors, lowest $P=0.23$; see also Figure 1-3). Analysis of each event relative to its immediately preceding baseline period confirmed that BLA neurons were robustly activated by CS onset as reflected in the peak calcium response $\left(\mathrm{CS}: F_{(1,10)}=7.25, P=0.02\right.$; Training: $F_{(2.5,24.5)}=1.88, P=0.17 ; \mathrm{CS} \times$ Training: $\left.F_{(1.2,12.4)}=0.54, P=0.51\right)$ and AUC (CS: $F_{(1,10)}=6.28, P=0.03$; Training: $F_{(1.9,19.3)}=0.40, P=0.67$; CS x Training: $\left.F_{(1.2,11.7)}=0.17, P=0.73\right)$, as well as at reward retrieval during the cue $\left[\left(\right.\right.$ Peak, Reward: $F_{(1,10)}=$ 16.82, $P=0.002$; Training: $F_{(1.9,19.4)}=3.41, P=0.06$; Reward $x$ Training: $\left.F_{(1.7,16.8)}=0.88, P=0.42\right)(A \cup C$, Reward: $F_{(1,10)}=15.21, P=0.003$; Training: $F_{(1.6,15.7)}=2.13, P=0.16$; Reward $x$ Training: $F_{(1.5,14.8)}=1.25, P=$ 0.30)]. The same BLA reward response could also be detected when the data were aligned to reward delivery (Figure 1-4). There were no significant BLA activity changes detected in response to food-port entries absent reward (Figure 1-5), indicating that reward retrieval responses resulted from reward experience rather than the act of entering the food port. Thus, BLA neurons are active at the most critical time for the encoding of stimulusoutcome memories, when the reward is experienced during the cue (i.e., the stimulus-outcome pairing).

It was surprising that responses to the cues were present on the first conditioning session, particularly in light of evidence that BLA responses to both appetitive and aversive cues increase across learning (Crouse et al., 2020; Johansen et al., 2010; Lutas et al., 2019; Tye et al., 2008). This could reflect a non-associative, novelty response to either or both the tone or white noise presentation. To examine this and, thus, evaluate whether the BLA cue responses later in training were due to stimulus-outcome learning, we repeated the experiment in a separate group of naïve rats, but this time omitted the reward delivery during the Pavlovian conditioning (Figure 2a-c; $N=6$ ). Instead, the rewards were delivered unpaired with the cues several hours after each session in a distinct context. Like presentation of the reward-predictive cues, presentation of either the tone or white noise stimulus unpaired with reward $\left(\mathrm{CS}_{\varnothing}\right)$ robustly activated BLA neurons during the first session, but, in contrast to the reward-predictive cues, this effect habituated over sessions (Figure 2d). Both tone and noise elicited a similar elevation in the peak calcium response that was largest on session 1 and diminished with subsequent days of exposure (Figure 2e; Session x CS $\varnothing: F_{(4,20)}=3.25, P=0.03$; $\mathrm{CS}_{\varnothing}$ presence: $F_{(0.4,2.1)}=4.84, P=0.13$; $\mathrm{CS}_{\varnothing}$ type (white noise v. tone): $F_{(0.3,1.5)}=7.03, P=0.12$; Session: $F_{(2.3,11.7)}=3.27, P=0.07$; Session $\times \mathrm{CS}_{\varnothing}$ type: $F_{(4,20)}=$ 1.42, $P=0.26 ; \mathrm{CS}_{\varnothing} \times \mathrm{CS}_{\varnothing}$ type: $F_{(0.5,2.3)}=9.69, P=0.07$; Session $\times \mathrm{CS}_{\varnothing} \times \mathrm{CS}_{\varnothing}$ type: $\left.F_{(0.6,3.2)}=0.80, P=0.37\right)$. 
The effect was similar when quantified using area under the calcium curve (Figure $2 \mathrm{f}$; Session $\times \mathrm{CS}_{\varnothing}$ : $F_{(4,20)}=$ 2.65, $P=0.06 ; \mathrm{CS}_{\varnothing}$ presence: $F_{(0.5,2.4)}=5.07, P=0.12 ; \mathrm{CS}_{\varnothing}$ type: $F_{(0.3,1.4)}=4.81, P=0.14 ;$ Session: $F_{(2.6,12.8)}=$ 1.55, $P=0.25$; Session $\times \mathrm{CS}_{\varnothing}$ type: $F_{(4,20)}=1.14, P=0.37 ; \mathrm{CS}_{\varnothing} \times \mathrm{CS}_{\varnothing}$ type: $F_{(0.5,2.4)}=10.43, P=0.06$; Session $\times \mathrm{CS}_{\varnothing} \times \mathrm{CS}_{\varnothing}$ type: $\left.F_{(0.7,3.7)}=1.81, P=0.24\right)$. To check whether the decline of the $\mathrm{CS}_{\varnothing}$ response was due simply to signal degradation over time, following the last $\mathrm{CS}_{\varnothing}$ session we recorded BLA calcium responses to unpredicted reward delivery. Rewards were capable of robustly activating the BLA (Figure $2 \mathrm{~g}-\mathrm{i}$; Peak, $\mathrm{t}_{5}=2.93$, $\left.P=0.03 ; \mathrm{AUC}, \mathrm{t}_{5}=4.07, P=0.01\right)$. This positive control indicates that the decline of the BLA CS $\varnothing$ response was due to stimulus habituation, not signal degradation. Thus, the BLA response to cue presentation during early Pavlovian conditioning likely reflects a non-associative novelty effect that habituates with subsequent exposure, indicating that the BLA responses to the reward-predictive cues later in training (Figure 1) largely result from the association with reward.

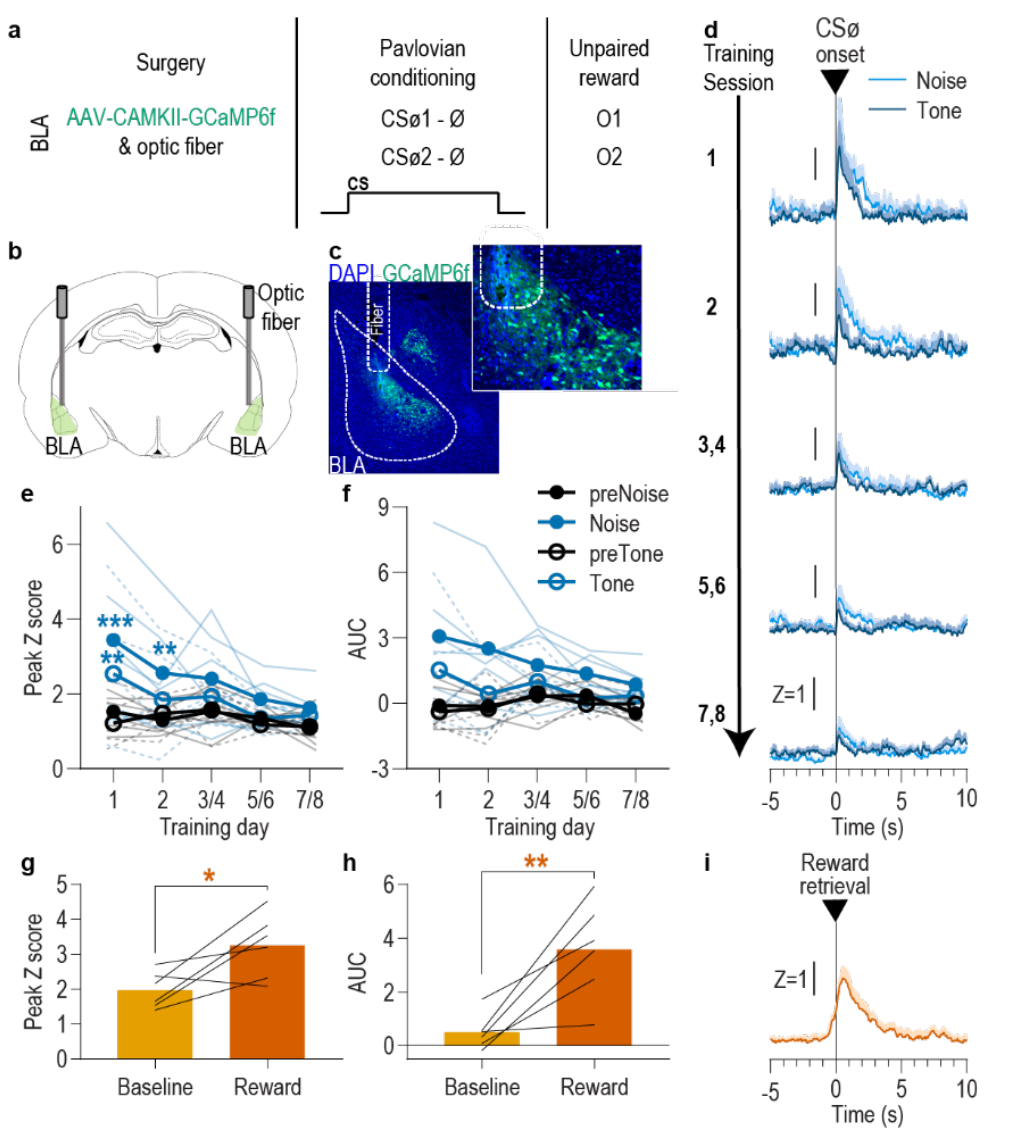

Figure 2. BLA neurons are only transiently activated by stimuli if they are not paired with reward. (a) Procedure schematic. CSø, neutral stimulus; $\varnothing$, no reward outcome; O, outcome (sucrose solution or food pellet). (b) Schematic of fiber photometry approach for imaging bulk calcium activity in BLA neurons. (c) Representative fluorescent image of GCaMP6f expression and fiber placement in the BLA. (d) Trial-averaged GCaMP6f fluorescence change (Z-scored $\Delta F / F)$ in response to noise and tone CSø onset across days. Shading reflects between-subjects s.e.m.. (e-f) Trial-averaged quantification of maximal (e; peak) and area under the GCaMP Z-scored $\Delta F / F$ curve (f; AUC) during the $3 \mathrm{~s}$ following noise and tone $\mathrm{CS} \varnothing$ onset compared to equivalent baseline periods immediately prior to each event. Thin light lines represent individual subjects (solid = Noise, dashed = Tone). (g-h) Trial-averaged quantification of maximal (g; peak) and area under the GCaMP Z-scored $\triangle F / F$ curve (h; AUC) during the $3 \mathrm{~s}$ following retrieval of the unpaired reward compared to equivalent baseline period immediately prior reward retrieval. Lines represent individual subjects. (i) Trial-averaged GCaMP6f fluorescence (Zscored $\Delta \mathrm{F} / \mathrm{F})$ in response to unpaired reward, averaged across reward type. Shading reflects between-subjects s.e.m.. $N=6$. ${ }^{*} P<0.05$, ${ }^{* *} P<.01$ relative to pre-event baseline.

\section{BLA neuron activity is necessary during outcome experience to encode appetitive Pavlovian stimulus- outcome memories.}


We found that BLA neurons are robustly activated at the time at which stimulus-reward memories can be formed: when the reward is experienced during a predictive cue. We next asked whether this activity is necessary for such learning and, if so, whether it is necessary for encoding sensory-specific stimulus-outcome memories (Figure 3a). We expressed the inhibitory opsin archaerhodopsin T (ArchT; $N=9$ ) or eYFP control $(N=10)$ in BLA, primarily, principal neurons (Figure $3 b-d)$ to allow green light $(532 \mathrm{~nm}, \sim 10 \mathrm{~mW})$ to transiently hyperpolarize and inhibit the activity of these cells (Figure 3-1). Rats were again given 8 Pavlovian conditioning sessions during which each of 2 distinct, 2-min auditory CSs was paired with intermittent delivery of one specific food reward (8 of each CS/session). During each Pavlovian conditioning session, we optically inhibited the activity of BLA neurons during each cue. We restricted inhibition to $5 \mathrm{~s}$ concurrent with the delivery and retrieval of each food reward because this is the time at which the stimulus-outcome pairing occurs and when we found the BLA to be endogenously active (Figure 1). Optical inhibition of BLA neurons at reward experience during Pavlovian conditioning did not impede the development of the Pavlovian conditional goal-approach response to the cue sampled prior to reward delivery (Figure 3e; Training: $F_{(3.8,64.9)}=17.53, P<0.0001$; Virus (eYFP v. ArchT): $F_{(1,17)}=0.19, P=0.67$; Virus $x$ Training: $F_{(7,119)}=1.28, P=0.26$; see also Figure 3-2a). This general conditional response at the shared food port, however, does not require that the subjects have learned the sensory-specific details of the predicted reward. To test for such stimulus-outcome memory encoding, we gave subjects instrumental conditioning followed by a PIT test. Both were conducted without any manipulation. During instrumental conditioning, rats were trained that two different actions (left or right lever press) each earned one of the unique food rewards (e.g., left press $\rightarrow$ sucrose/right press $\rightarrow$ pellets; Figure 3-2b). At the PIT test both levers

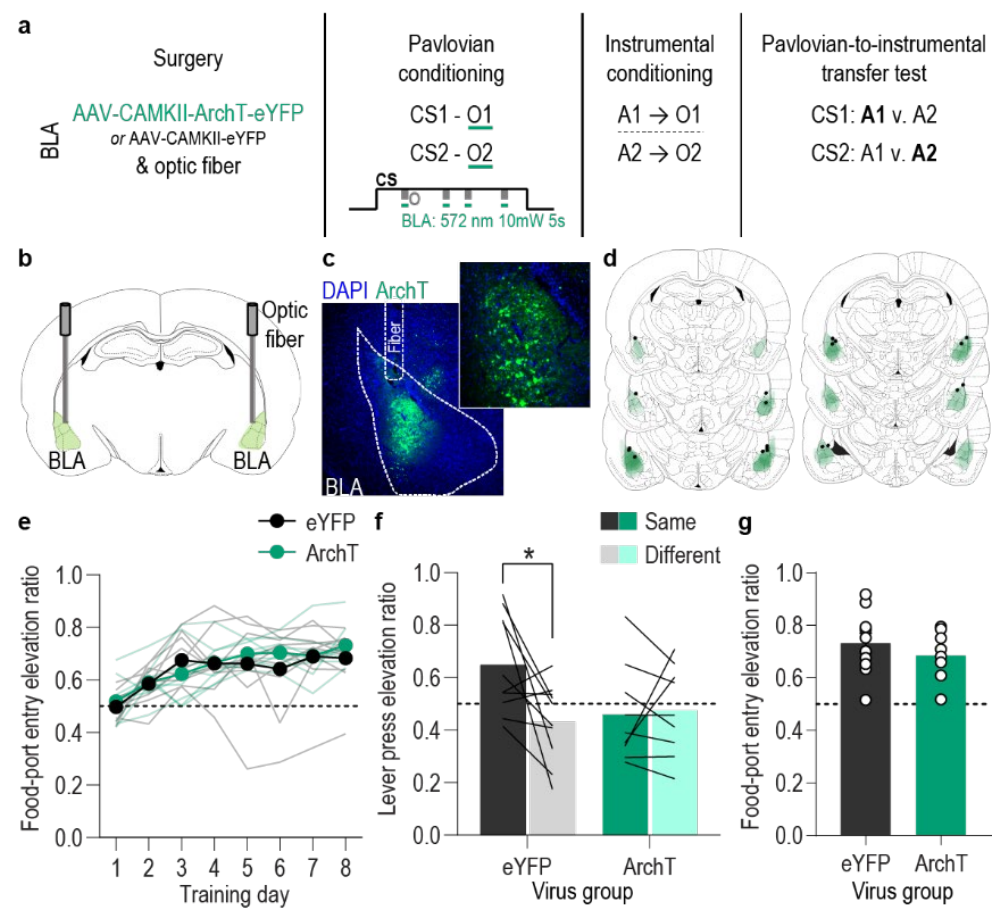

Figure 3. Optical inhibition of BLA neurons during stimulus-outcome pairing attenuates the encoding of stimulus-outcome memories. (a) Procedure schematic. CS, conditional stimulus (white noise or tone); O, outcome (sucrose solution or food pellet); A, action (left or right lever press). (b) Schematic of optogenetic strategy for bilateral inhibition of BLA neurons. (c) Representative fluorescent image of ArchT-eYFP expression and fiber placement in the BLA. (d) Schematic representation of ArchT-eYFP expression and placement of optical fiber tips in BLA for all subjects. (e) Elevation [(CS probe entry rate)/(CS probe entry rate + preCS entry rate)] in food-port entries during the CS probe period (after CS onset, before first reward delivery), averaged across trials and CSs for each day of Pavlovian conditioning. Thin light lines represent individual subjects. (f) Elevation in lever presses on the lever that earned the same outcome as the presented CS (Same; [(presses on Same lever during CS)/(presses on Same lever during CS + Same presses during preCS)], averaged across trials and across CSs), relative to the elevation in responding on the alternate lever (Different; [(presses on Different lever during CS)/(presses on Different lever during CS + Different presses during preCS)], averaged across trials and across CSs) during the PIT test. Lines represent individual subjects. (g) Elevation in food-port entries to CS presentation (averaged across trials and CSs) during the PIT test. Circles represent individual subjects. ArchT, $N=9$; eYFP, $N=10 .{ }^{*} P<0.05$. 
were present, but lever pressing was not rewarded. Each CS was presented 4 times (also without accompanying reward), with intervening CS-free baseline periods, to assess its influence on action performance and selection in the novel choice scenario. Because the cues are never associated with the instrumental actions, this test assesses the ability to, upon cue presentation, retrieve a memory of the specific predicted reward and use it to motivate choice of the action known to earn the same unique reward (Colwill \& Motzkin, 1994; Corbit \& Balleine, 2016; Gilroy et al., 2014; Kruse et al., 1983). If subjects had encoded detailed stimulus-outcome memories during Pavlovian conditioning, then the CS should cause them to increase presses selectively on the lever that, during training, earned the same outcome as predicted by that cue. Controls showed this outcome-specific PIT effect (Figure 3f). Conversely, the cues were not capable of influencing lever-press choice in the group for which the BLA was inhibited at the time of outcome experience during Pavlovian conditioning (Figure 3f; Virus $x$ Lever: $F_{(1,17)}=5.10, P=0.04$; Virus: $F_{(1,17)}=1.41, P=0.25$; Lever (Same v. Different): $F_{(1,17)}=3.84, P=0.07$; see also Figure 3-2c). As in training, during this PIT test the conditional goal-approach response was similar between groups (Figure $3 g ; t_{17}=0.94, P=0.36$; see also Figure $3-2 d$ ). Thus, BLA neuronal activity is not needed for the learning that supports general conditional approach responses, but is necessary, specifically at the time of outcome experience, to link the sensory-specific details of the outcome to a predictive cue. Such encoding is critical for that cue to subsequently guide decision making.

An alternative possibility is that the total amount of inhibition compromised BLA activity more broadly. That is, that BLA activity per se rather than specifically at the time of stimulus-outcome pairing mediates the encoding of stimulus-outcome memories. To rule this out, we repeated the experiment in a new cohort of naïve rats in which we matched the frequency and duration of inhibition to the experimental group, but delivered it during baseline pre-CS periods during Pavlovian conditioning. This inhibition had no effect on the subsequent influence of the cues on instrumental choice behavior during the PIT test (Figure 3-3), confirming that BLA activity specifically at the time of stimulus-outcome pairing mediates the encoding of detailed stimulus-outcome memories.

\section{IOFC $\rightarrow$ BLA projections are necessary for encoding Pavlovian stimulus-outcome memories.}

We found that activity in BLA neurons at the time of reward delivery/experience mediates encoding of the relationship between that specific rewarding event and the environmental stimulus that predicts it. We next asked which BLA input might facilitate this function. The orbitofrontal cortex (OFC) is a prime candidate. The OFC sends dense glutamatergic innervation to the BLA (Aggleton et al., 1980; Carmichael \& Price, 1995; Heilbronner et al., 2016; Lichtenberg et al., 2017; Malvaez et al., 2019; Price, 2007) and is itself implicated in appetitive learning (Baltz et al., 2018; Murray \& Izquierdo, 2007; Ostlund \& Balleine, 2007b; Rudebeck \& Rich, 2018). BLA inputs from the lateral (IOFC), rather than medial OFC subregion, have previously been shown to be involved in learning information about a reward (i.e., its incentive value) (Malvaez et al., 2019), but are not required for retrieving appetitive memories (Lichtenberg et al., 2017; Malvaez et al., 2019). Thus, this pathway might play a critical role specifically in forming stimulus-outcome associative memories. To evaluate this, we used pathwayspecific optical inhibition to ask whether activity in IOFC $\rightarrow$ BLA projections mediates the encoding of stimulusoutcome memories (Figure 4a). We expressed ArchT $(N=8)$ or eYFP control $(N=8)$ in IOFC neurons and detected expression in IOFC axons and terminals in the BLA in the vicinity of implanted optical fibers (Figure 4bd). Green light $(532 \mathrm{~nm}, \sim 10 \mathrm{~mW})$ was used to inhibit IOFC axons and terminals in the BLA (Figure 4-1). Subjects received Pavlovian conditioning, as above, and inhibition was again restricted to $5 \mathrm{~s}$ during the delivery and retrieval of each reward during each cue. Similar to inhibition of BLA neurons, optical inhibition of IOFC $\rightarrow B L A$ projection activity during stimulus-outcome pairing did not affect the development of the Pavlovian conditional goal-approach response (Figure 4e; Training: $F_{(3.9,54.3)}=7.84, P<0.0001$; Virus: $F_{(1,14)}=0.22, P=0.65$; Virus $\mathrm{x}$ Training: $F_{(7,98)}=0.43, P=0.88$; see also Figure 4-2a) or its expression during the PIT test (Figure $4 \mathrm{~g}$; $t_{14}=$ $0.49, P=0.63$; see also Figure 4-2d). It did, however, attenuate encoding of sensory-specific stimulus-outcome 
memories as evidenced by the subjects' inability to later use those memories to allow cue presentation to bias choice behavior during the PIT test (Figure 4f; Virus $x$ Lever: $F_{(1,14)}=6.49, P=0.02$; Virus: $F_{(1,14)}=0.04, P=$ 0.85 ; Lever: $F_{(1,14)}=7.10, P=0.02$; see also Figure $\left.4-2 \mathrm{c}\right)$. Thus, activity in IOFC $\rightarrow$ BLA projections regulates the encoding of detailed, sensory-specific stimulus-outcome memories. Together, with prior evidence that inactivation of IOFC $\rightarrow$ BLA projections does not disrupt the expression of outcome-selective PIT (Lichtenberg et al., 2017), these data suggest that activity in IOFC $\rightarrow$ BLA projections mediates the encoding, but not retrieval of stimulus-outcome memories.

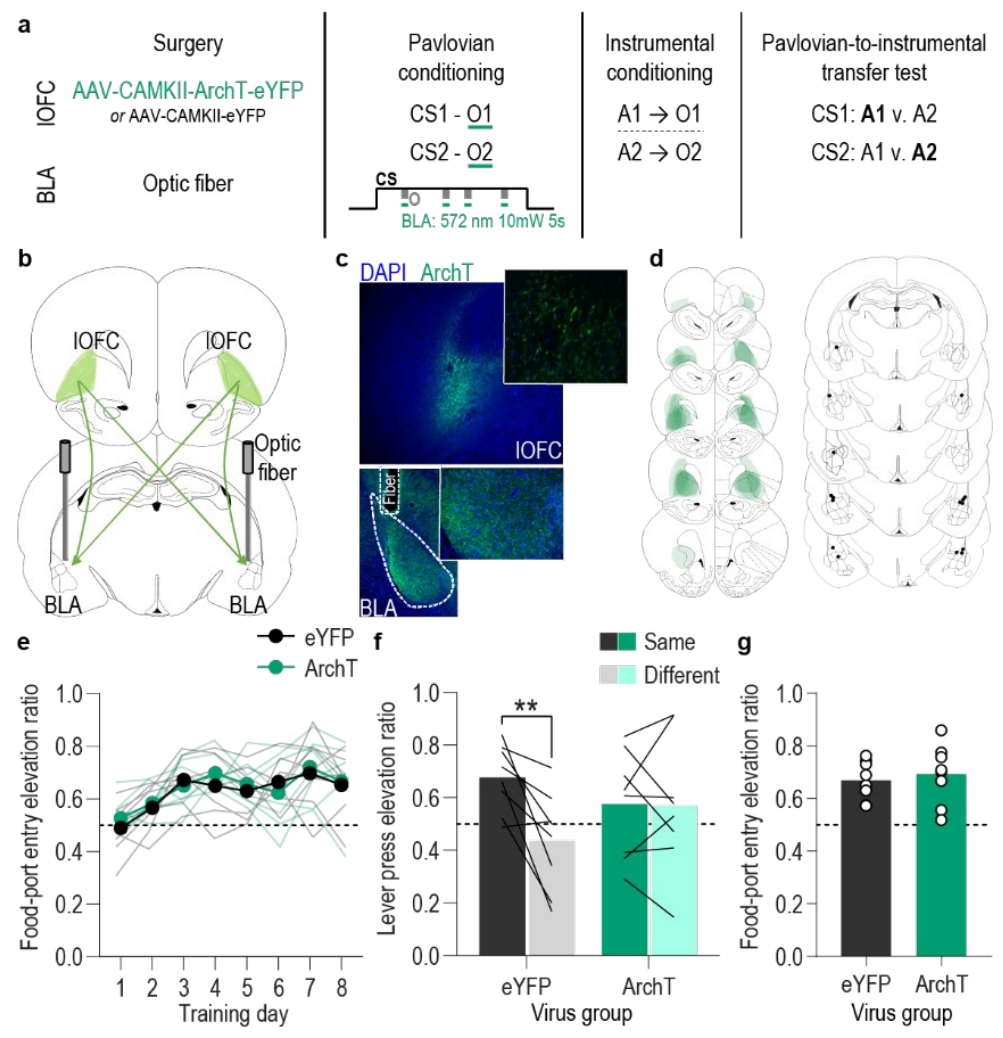

Figure 4. Optical inhibition of IOFC terminals in the BLA during stimulus-outcome pairing attenuates the encoding of stimulusoutcome memories. (a) Procedure schematic. CS, conditional stimulus (white noise or tone); O, outcome (sucrose solution or food pellet); A, action (left or right lever press). (b) Schematic of optogenetic strategy for bilateral inhibition of IOFC axons and terminals in the BLA. (c) Top: Representative fluorescent image of ArchT-eYFP expression in IOFC cell bodies. Bottom: Representative image of fiber placement in the vicinity of immunofluorescent ArchT-eYFP-expressing IOFC axons and terminals in the BLA. (d) Schematic representation of ArchT-eYFP expression in IOFC and placement of optical fiber tips in BLA for all subjects. (e) Elevation [(CS probe entry rate)/(CS probe entry rate + preCS entry rate)] in food-port entries during the CS probe period (after CS onset, before first reward delivery), averaged across trials and CSs for each day of Pavlovian conditioning. Thin light lines represent individual subjects. (f) Elevation in lever presses on the lever that earned the same outcome as the presented CS (Same; [(presses on Same lever during CS)/(presses on Same lever during CS + Same presses during preCS)], averaged across trials and across CSs), relative to the elevation in responding on the alternate lever (Different; [(presses on Different lever during CS)/(presses on Different lever during CS + Different presses during preCS)], averaged across trials and across CSs) during the PIT test. Lines represent individual subjects. (g) Elevation in food-port entries to CS presentation (averaged across trials and CSs) during the PIT test. Circles represent individual subjects. ArchT, $N=8 ;$ eYFP, $N=$ 8. ${ }^{* *} P<0.01$.

\section{$I O F C \rightarrow B L A \rightarrow I O F C$ is a stimulus-outcome memory circuit.}

Collectively, the data show that the BLA, with help from direct IOFC input, mediates the encoding of the detailed cue-reward memories that enable the cues to trigger the sensory-specific reward outcome representations that influence decision making. The IOFC-BLA circuit is bidirectional. The BLA sends dense excitatory projections back to the IOFC (Barreiros et al., 2021; Lichtenberg et al., 2017; Morecraft et al., 1992). Activity in these projections mediates the representation of expected outcomes in the IOFC (Rudebeck et al., 2013; Rudebeck et al., 2017; Schoenbaum et al., 2003) and the use of stimulus-outcome memories to guide choice (Lichtenberg et 
al., 2017). But it remains unknown whether BLA $\rightarrow$ IOFC projection activity enables the use of the associative information that is learned via activation of IOFC $\rightarrow$ BLA projections. That is, whether IOFC $\rightarrow B L A \rightarrow I O F C$ is a functional stimulus-outcome memory encoding and retrieval circuit or whether IOFC $\rightarrow$ BLA and BLA $\rightarrow$ IOFC projections tap in to independent, parallel information streams. Indeed, stimulus-outcome memories are highly complex including multifaceted information about outcome attributes (e.g., value, taste, texture, nutritional content, category, probability, timing, etc.) and related consummatory and appetitive responses (Delamater \& Oakeshott, 2007). Therefore, we next asked whether the IOFC $\rightarrow$ BLA and BLA $\rightarrow$ IOFC pathways form a functional stimulus-outcome memory encoding and retrieval circuit, i.e., whether the sensory-specific associative information that requires IOFC $\rightarrow B L A$ projections to be encoded also requires activation of BLA $\rightarrow$ IOFC projections to be used to guide decision making, or whether these are independent, parallel pathways, tapping into essential but independent streams of information.

To arbitrate between these possibilities, we multiplexed optogenetic and chemogenetic inhibition to perform a serial circuit disconnection. We disconnected IOFC $\rightarrow$ BLA projection activity during stimulus-outcome learning from BLA $\rightarrow$ IOFC projection activity during the retrieval of these memories at the PIT test (Figure 5a). For the disconnection group $(N=10)$, we again expressed ArchT bilaterally in IOFC neurons (Figure $5 \mathrm{~b}$-d) to allow expression in IOFC axons and terminals in the BLA. This time, we implanted the optical fiber only unilaterally in the BLA (Figure $5 \mathrm{~b}-\mathrm{d})$, so that green light $(532 \mathrm{~nm}, \sim 10 \mathrm{~mW})$, delivered again during Pavlovian conditioning for 5 $s$ during the delivery and retrieval of each reward during each cue, would inhibit both the ipsilateral and contralateral IOFC input to the BLA of only one hemisphere. In these subjects, we also expressed the inhibitory designer receptor human M4 muscarinic receptor (hM4Di) unilaterally in the BLA of the hemisphere opposite to the optical fiber and in that same hemisphere placed a guide cannula over the IOFC near hM4Di-expressing BLA axons and terminals (Figure $5 \mathrm{~b}-\mathrm{d}$ ). This allowed us to infuse the hM4Di ligand clozapine- $n$-oxide (CNO; $1 \mathrm{mM}$ in $0.25 \mu \mathrm{l})$ prior to the PIT test to unilaterally inhibit BLA terminals in the IOFC, which are largely ipsilateral (Lichtenberg et al., 2017), in the hemisphere opposite to that for which we had inhibited IOFC $\rightarrow$ BLA projection activity during Pavlovian conditioning. Thus, we optically inhibited the IOFC $\rightarrow$ BLA stimulus-outcome learning pathway in one hemisphere at each stimulus-outcome pairing during Pavlovian conditioning, and chemogenetically inhibited the putative BLA $\rightarrow$ IOFC retrieval pathway in the opposite hemisphere during the PIT test in which stimulus-outcome memories must be used to guide choice. If BLA $\rightarrow$ IOFC projection activity mediates the retrieval of the sensory-specific associative memory that requires activation of IOFC $\rightarrow B L A$ projections to be encoded, then we will have bilaterally disconnected the circuit, attenuating encoding in one hemisphere and retrieval in the other, thereby disrupting the ability to use the stimulus-outcome memories to guide choice behavior during the PIT test. If, however, these pathways mediate parallel information streams, i.e., independent components of the stimulus-outcome memory, the expression of PIT should be intact because one of each pathway is undisrupted to mediate its individual component during each phase. The control group received identical procedures with the exception that viruses lacked ArchT and hM4Di $(N=8)$. To control for unilateral inhibition of each pathway without disconnecting the circuit, a second control group $(N=8)$ received the same procedures as the experimental contralateral ArchT/hM4Di disconnection group, except with BLA hM4Di and the IOFC guide cannula in the same hemisphere as the optical fiber used to inactivate IOFC axons and terminals in the BLA (Figure 5-1). Thus, during the PIT test for this group the BLA $\rightarrow I O F C$ pathway was chemogenetically inactivated in the same hemisphere in which the IOFC $\rightarrow$ BLA pathway had been optically inactivated during Pavlovian conditioning, leaving the entire circuit undisrupted in the other hemisphere. These control groups did not differ on any measure and so were collapsed into a single control group [(Pavlovian training, Training: $F_{(2.2,31.3)}=12.96, P<0.0001$; Control group type: $F_{(1,14)}=0.02, P=0.89$; Group $x$ Training: $\left.F_{(7.98)}=0.76, P=0.62\right)\left(\right.$ PIT Lever presses, Lever: $F_{(1,14)}=14.68, P=0.002$; Control group type: $F_{(1,14)}=0.38, P=$ 0.55; Group x Lever: $F_{(1,14)}=0.43, P=0.52$ ) (PIT Food-port entries, $t_{14}=0.72, P=0.48$ )]. See also Figure 5-2 for disaggregated control data. 
We found evidence that activity in IOFC $\rightarrow$ BLA projections mediates the encoding of the sensory-specific stimulus-outcome memory that is later used to allow cues to guide choice via activation of BLA $\rightarrow$ IOFC projections. As with the bilateral inhibition experiments, the control and disconnection groups both developed a Pavlovian conditional goal-approach response with training (Figure 5e; Training: $F_{(2.8,68.1)}=28.13, P<0.0001$; Group (Combined control group v. Contralateral ArchT/hM4Di- disconnection): $F_{(1,24)}=0.46, P=0.51$; Group $\mathrm{x}$ Training: $F_{(7,168)}=0.44, P=0.88$; see also Figure $\left.5-2 \mathrm{a}\right)$, which was similarly expressed during the PIT test (Figure $5 \mathrm{~g} ; t_{24}=0.11, P=0.91$; see also Figure 5-2d). But disconnection of IOFC $\rightarrow$ BLA projection activity during stimulus-outcome learning from BLA $\rightarrow$ IOFC projection activity during the PIT test attenuated the ability to use such memories to guide choice behavior (Figure 5f; Group x Lever: $F_{(1,24)}=5.57, P=0.03$; Group: $F_{(1,24)}=$ $0.47, P=0.50$; Lever: $F_{(1,24)}=1.39, P=0.21$; see also Figure $\left.5-2 \mathrm{c}\right)$. Whereas in the control group cue presentation significantly biased choice towards the action earning the same predicted reward, this outcomespecific PIT effect did not occur in the disconnection group. Rather, during the cues rats in the disconnection group showed a non-discriminate elevation in pressing on both levers (Figure 5-2c). Thus, disconnection of IOFC $\rightarrow$ BLA projection activity during stimulus-outcome learning from BLA $\rightarrow$ IOFC projection activity during the retrieval of this information attenuated the ability to use stimulus-outcome memories to bias choice behavior, indicating that the IOFC and BLA form a bidirectional circuit for the encoding (IOFC $\rightarrow$ BLA) and use (BLA $\rightarrow$ IOFC) of appetitive stimulus-outcome memories.

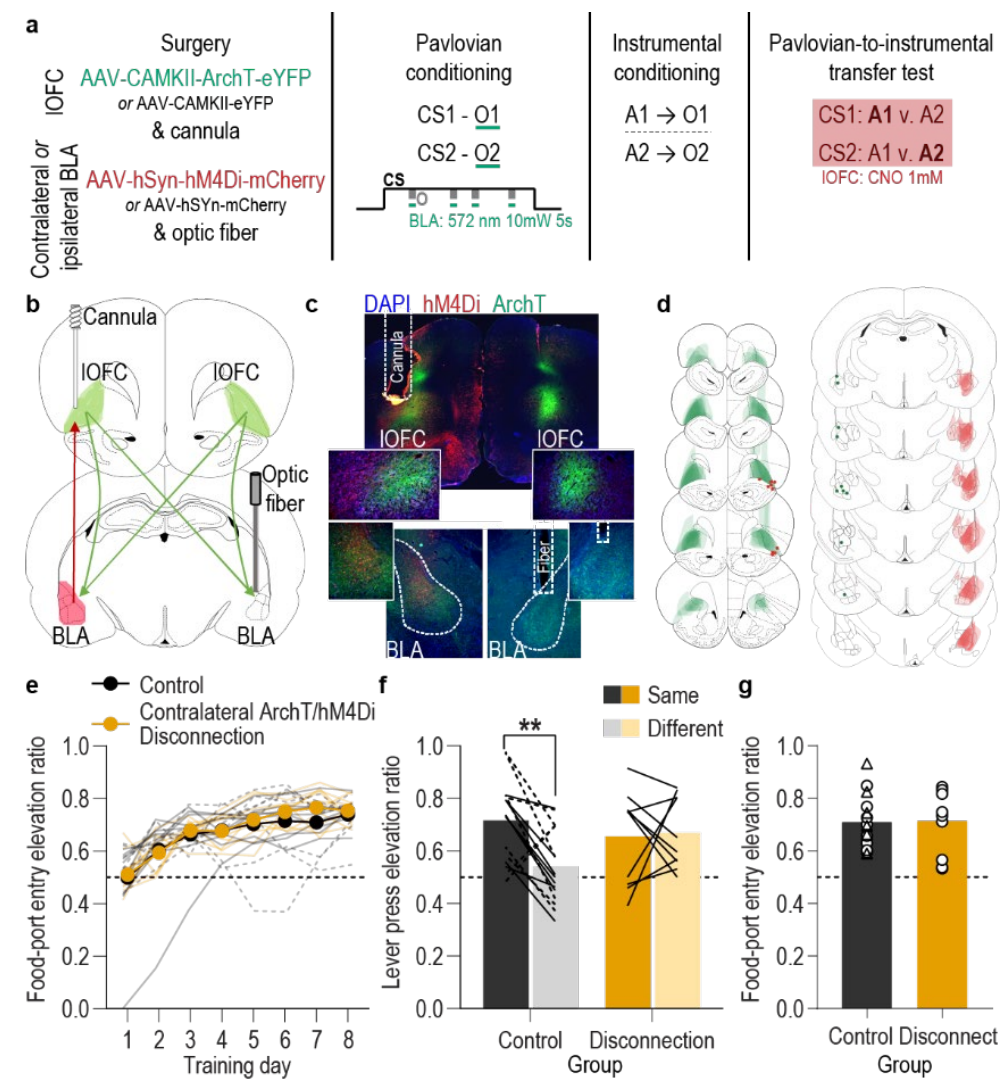

Figure 5. Serial disconnection of IOFC $\rightarrow$ BLA projections during stimulus-outcome pairing from BLA $\rightarrow$ IOFC projections during Pavlovian-to-instrumental transfer test disrupts stimulus-outcome memory. (a) Procedure schematic. CS, conditional stimulus (white noise or tone); O, outcome (sucrose solution or food pellet); A, action (left or right lever press); CNO, clozapine- $n$-oxide. (b) Schematic of multiplexed optogenetic/chemogenetic inhibition strategy for unilateral optical inhibition of IOFC $\rightarrow$ BLA projections during Pavlovian conditioning and contralateral, unilateral, chemogenetic inhibition of BLA $\rightarrow$ IOFC projections during the PIT test. (c) Top: Representative fluorescent image of bilateral ArchT-eYFP expression in IOFC cells bodies and unilateral expression of hM4Di-mCherry in BLA axons and terminals in the IOFC in the vicinity of implanted guide cannula. Bottom: Representative image of unilateral BLA fiber placement in the vicinity of immunofluorescent ArchT-eYFP expressing IOFC axons and terminals (right) and unilateral expression of hM4Di-mCherry in BLA cell bodies in the contralateral hemisphere (left). (d) Schematic representation of bilateral ArchT-eYFP expression and unilateral cannula placement in IOFC and unilateral hM4Di expression and placement of optical fiber tips in the contralateral BLA for 
all Contralateral group subjects. Fibers are shown in left and cannula placement in the right hemisphere, but fiber/cannula hemisphere arrangement was counterbalanced across subjects. See Figure 5-1 for histological verification of ipsilateral control. (e) Elevation [(CS probe entry rate)/(CS probe entry rate + preCS entry rate)] in food-port entries during the CS probe period (after CS onset, before first reward delivery), averaged across trials and CSs for each day of Pavlovian conditioning. Thin light lines represent individual subjects (Contralateral eYFP/mCherry (solid lines) and Ipsilateral ArchT/hM4Di (dashed lines) collapsed into a single control group). (f) Elevation in lever presses on the lever that earned the same outcome as the presented CS (Same; [(presses on Same lever during CS)/(presses on Same lever during CS + Same presses during preCS)], averaged across trials and across CSs), relative to the elevation in responding on the alternate lever (Different; [(presses on Different lever during CS)/(presses on Different lever during CS + Different presses during preCS)], averaged across trials and across CSs) during the PIT test. Lines represent individual subjects. (g) Elevation in food-port entries to CS presentation (averaged across trials and CSs) during the PIT test. Data points represent individual subjects, triangles indicate ipsilateral control subjects. Control, $N=16$; Contralateral disconnection group, $N=10 .{ }^{* *} P<0.01$.

\section{DISCUSSION}

Using fiber photometry bulk calcium imaging, cell-type and pathway-specific optogenetic inhibition, multiplexed optogenetic and chemogenetic inhibition, Pavlovian conditioning, and the outcome selective PIT test, we explored the function of the BLA and its interaction with the IOFC in the ability to learn detailed cue-reward memories and use them to guide decision making. Such memories are critical to the ability to use environmental cues to infer which specific rewards are likely to be available in the current state and, thus, to choose adaptively. We found that the BLA is robustly activated at the time of stimulus-outcome pairing and that this activity is necessary for sensory-specific, appetitive associative memories to be encoded, so that they can later influence decision making. We also found that this BLA activity is not necessary for the appetitive learning that supports general conditional goal-approach behavior, which does not require a detailed stimulus-outcome memory. IOFC input to the BLA supports its function in encoding stimulus-outcome memories and BLA projections back to the IOFC mediate the use of these memories to guide decision making. Thus, the IOFC $\rightarrow$ BLA $\rightarrow$ IOFC circuit regulates the encoding and subsequent use of the state-dependent and sensory-specific reward memories that are critical for decision making between two appetitive choices.

BLA neurons were found to be robustly activated at the time of stimulus-reward pairing as well as at cue onset, consistent with prior evidence that the BLA is activated by both rewards (Crouse et al., 2020; Fontanini et al., 2009; Malvaez et al., 2019; Roesch et al., 2010; Schoenbaum et al., 1998a; Sugase-Miyamoto \& Richmond, 2005) and their predictors (Belova et al., 2008; Beyeler et al., 2018; Beyeler et al., 2016; Crouse et al., 2020; Lutas et al., 2019; Malvaez et al., 2015; Muramoto et al., 1993; Paton et al., 2006; Schoenbaum et al., 1998a, 1999; Sugase-Miyamoto \& Richmond, 2005; Tye \& Janak, 2007; Tye et al., 2008). Interestingly, the cues triggered a transient elevation in BLA activity at their onset, rather than a sustained elevation throughout their 2min duration, perhaps suggesting that such activity reflects the state change, rather than the state per se. Both the cue and reward responses were present from the first conditioning session and persisted throughout training. That we detected cue responses on the first day of training before associative learning had occurred is, perhaps, unexpected and likely due to the novelty of the auditory stimuli during early training (Bordi \& LeDoux, 1992; Bordi et al., 1993; Cromwell et al., 2005; Romanski et al., 1993). Indeed, we found that presentation of identical auditory stimuli unpaired with reward activated BLA neurons during the first session, much like the reward-predictive cues, but, in contrast to the reward-predictive cues, this response habituated over subsequent sessions. Thus, BLA cue responses later in training result from appetitive associative learning. Whereas we detected reward responses throughout training, prior data have demonstrated a shift in BLA responses from the reward to predictive events (Crouse et al., 2020) and little response to rewards in the absence of learning (Malvaez et al., 2015). The persistent reward response detected here likely results from the uncertainty of reward timing during the cues, which set the context for the intermittent availability of one specific reward. Another possibility is that it relates to the learning of two unique cue-reward contingencies, which was not the case in prior tasks. Nonetheless, the data show the BLA to be robustly activated at the time of stimulus-reward pairing in a task known to engender the encoding of detailed, sensory-specific stimulus-outcome memories. 
We also found the BLA to be necessary, specifically at the time of stimulus-reward pairing, to encode the detailed stimulus-outcome memories. This is consistent with evidence that either pre- or post-training BLA lesion or pre-test inactivation disrupts appetitive conditional behaviors that rely on a sensory-specific, stimulus-outcome memory in rodents (Blundell et al., 2001; Corbit \& Balleine, 2005; Derman et al., 2020; Hatfield et al., 1996; Lichtenberg et al., 2017; Lichtenberg \& Wassum, 2016; Malvaez et al., 2015; Morse et al., 2020; Ostlund \& Balleine, 2008) and in primates (Murray \& Izquierdo, 2007; Málková et al., 1997). Leveraging the temporal resolution of optogenetics, we demonstrated that BLA principal neurons mediate the encoding of such memories, and specifically that activity at the time of reward experience during a cue is critical. Inhibiting the BLA during reward experience attenuated the animal's ability to link that specific rewarding event to the associated cue, disrupting the encoding of the sensory-specific stimulus-outcome memories to the extent that animals were unable to later use those memories to guide choice behavior. Future work is needed to reveal the precise information content encoded by BLA neurons during reward experience that confers their necessary function in the formation of stimulus-outcome memories, though BLA neurons will respond selectively to unique food rewards (Liu et al., 2018), which could support the generation of sensory-specific reward memories. Whether BLA cue responses are also important for encoding stimulus-outcome memories is another important question exposed by the current results.

Although BLA activity during stimulus-outcome pairing was critical for encoding a detailed, outcome-specific, appetitive cue-reward memory, it was not necessary for the learning underlying the development a non-specific Pavlovian conditional goal-approach response, consistent with data collected with BLA lesions or inactivation (Corbit \& Balleine, 2005; Everitt et al., 2000; Hatfield et al., 1996; Malvaez et al., 2015; Morse et al., 2020; Parkinson et al., 2000). Although influenced by positive outcome valence, such responses do not require a rich sensory-specific representation of the predicted reward. Thus, BLA neurons appear not to be required to reinforce an appetitive Pavlovian response policy. Rather, the BLA mediates the encoding of the association between a cue and the specific reward it predicts, which includes encoding of the sensory-specific features of the reward. Optical stimulation of BLA neurons will, however, augment conditional goal-approach responses (Servonnet et al., 2020), suggesting BLA activation is capable of influencing such appetitive conditional behaviors.

Input from the IOFC was found to facilitate the BLA's function in mediating the encoding of stimulus-outcome memories. This expands upon previous findings that pre-training IOFC lesions disrupt behaviors that require a sensory-specific stimulus-outcome memory (Izquierdo et al., 2004; Machado \& Bachevalier, 2007; Ostlund \& Balleine, 2007a; Pickens et al., 2005; Pickens et al., 2003; Rhodes \& Murray, 2013; Scarlet et al., 2012), that the IOFC is active during cue-reward learning (Constantinople et al., 2019; Miller et al., 2018; Paton et al., 2006; Schoenbaum et al., 1998b; Takahashi et al., 2013; Wallis \& Miller, 2003), and that encoding of expected outcomes in the BLA requires an intact IOFC (Lucantonio et al., 2015; Saddoris et al., 2005). Our data add to this literature by revealing the causal contribution of the direct IOFC $\rightarrow$ BLA pathway, specifically at the time of stimulus-outcome pairing, to the formation of detailed, outcome-specific, appetitive associative memories. Indeed, IOFC neurons respond to rewarding events during learning to signal reward expectations that may support learning in downstream structures, such as the BLA (Stalnaker et al., 2007; Stalnaker et al., 2018). Prior evidence also indicates that activity in IOFC $\rightarrow$ BLA projections drives the encoding of the incentive value of a specific rewarding event (Malvaez et al., 2019). Such incentive value is dependent upon one's current physiological state (e.g., food has high value when hungry, but low when sated). Thus, IOFC $\rightarrow$ BLA projections may be responsible for linking states, defined by internal physiological and external predictive cues, to the specific rewarding events with which they are associated. The precise information content conveyed by IOFC $\rightarrow$ BLA projections and how it is used in the BLA is a critical question for follow-up investigation.

We also discovered that the IOFC and BLA form a bidirectional circuit for the encoding and use of appetitive stimulus-outcome memories. The BLA has been implicated in appetitive decision making (Costa et al., 2016; 
Costa et al., 2019; Izquierdo et al., 2013; Johnson et al., 2009; Orsini et al., 2017; Ostlund \& Balleine, 2008; Stolyarova et al., 2019; Wellman et al., 2005) and has been shown in non-human primates to interact with the IOFC in that regard (Baxter et al., 2000; Fiuzat et al., 2017). We previously found that BLA activity correlates with and regulates the ability to use sensory-specific, appetitive, stimulus-outcome memories to guide choice behavior (Malvaez et al., 2015). This function is mediated via direct BLA $\rightarrow$ IOFC projections, but does not require activation of IOFC $\rightarrow$ BLA projections (Lichtenberg et al., 2017). Here, using a serial disconnection procedure, we found that during reward choice BLA $\rightarrow$ IOFC projection activity mediates the use of the sensory-specific associative information that is learned via activation of IOFC $\rightarrow$ BLA projections. Thus, IOFC $\rightarrow B L A \rightarrow I O F C$ is a functional circuit for the encoding (IOFC $\rightarrow B L A)$ and subsequent use (BLA $\rightarrow$ IOFC) of sensory-specific reward memories to inform decision making. Interestingly, the serial disconnection disrupted the outcome-specificity of PIT but, unlike bilateral BLA or IOFC $\rightarrow$ BLA inhibition during learning, allowed the cues to non-discriminately excite instrumental activity. This could have resulted from incomplete disconnection. But it may indicate that IOFC $\rightarrow$ BLA projections facilitate the encoding of a broader set of information than that being transmitted back to the IOFC by BLA $\rightarrow$ IOFC projection activity during choice. BLA $\rightarrow$ IOFC projections mediate use of the sensoryspecific components of the reward memory needed to allow animals to know during a cue which specific reward is predicted and thus which action to select, but IOFC $\rightarrow$ BLA projections may facilitate the encoding of additional features of the memory, including those capable of promoting food- or reward-seeking activity more broadly. The encoding of such information would have been disrupted by bilateral IOFC $\rightarrow$ BLA or BLA inactivation during learning, but in the disconnection experiment could have been learned in the hemisphere that did not receive IOFC $\rightarrow$ BLA inactivation and subsequently retrieved via an alternate BLA pathway. Indeed, BLA $\rightarrow$ IOFC are not the only amygdala projections involved in reward memory (Beyeler et al., 2016; Corbit et al., 2013; Fisher et al., 2020; Kochli et al., 2020; Morse et al., 2020; Parkes \& Balleine, 2013).

IOFC activity in both humans and non-human animals can encode the features of an expected reward (Howard et al., 2015; Howard \& Kahnt, 2018; Klein-Flügge et al., 2013; Lopatina et al., 2015; McDannald et al., 2014; Pritchard et al., 2005; Suzuki et al., 2017; van Duuren et al., 2007; Zhou et al., 2019) and the IOFC has been proposed to be critical for using this information to guide decision making (Bradfield \& Hart, 2020; Delamater, 2007; Groman et al., 2019; Keiflin et al., 2013; Rich \& Wallis, 2016; Rudebeck \& Rich, 2018; Rudebeck \& Murray, 2014; Sharpe \& Schoenbaum, 2016; Wilson et al., 2014). This might especially be the case in novel situations (Gardner \& Schoenbaum, 2020). The PIT test is a novel choice scenario in which the subjects must use the cues to represent the sensory-specific features of the predicted reward, infer which reward is most likely to be available and, therefore, which action will be the most beneficial. IOFC $\rightarrow$ BLA projection activity, perhaps via relaying reward expectation (Stalnaker et al., 2007; Stalnaker et al., 2018), regulates the associative learning that may allow subsequent activity in BLA $\rightarrow$ IOFC projections to promote the representation of a specific predicted reward in the IOFC to enable decision making. The precise information content conveyed by each component of the IOFC-BLA circuit and how it is used in the receiving structure is a critical follow-up question that will require a cellular resolution investigation of the activity of each pathway. Another critical question is whether this circuitry similarly mediates appetitive associative learning and its influence on decision making in females. The exclusion of female subjects is a clear limitation of this study, though females do show similar performance in the task used here and also require the BLA and IOFC for its performance (Ostlund \& Balleine, $2007 a, 2008)$. Whether this IOFC-BLA architecture also underlies sensory-specific aversive memory is also a question ripe for further exploration.

The BLA, via input from the IOFC, helps to link environmental cues to the specific rewards they predict and, via projections back to the IOFC, to allow the cues to access those representations to influence decision making. An inability to either properly encode reward memories or to use such memories to inform decision making can lead to ill-informed motivations and decisions. This is characteristic of the cognitive symptoms underlying many psychiatric diseases, including substance use disorder. The OFC-BLA circuit is known to be altered by addictive 
bioRxiv preprint doi: https://doi.org/10.1101/2021.03.20.436233; this version posted June 9, 2021. The copyright holder for this preprint (which was not certified by peer review) is the author/funder, who has granted bioRxiv a license to display the preprint in perpetuity. It is made available under aCC-BY-NC-ND 4.0 International license.

Sias et al. 15

substances (Arguello et al., 2017) and to be dysfunctional in myriad psychiatric illnesses (Goldstein \& Volkow, 2011; Liu et al., 2014; Passamonti et al., 2012; Ressler \& Mayberg, 2007; Sladky et al., 2015). Thus, these data may also aid our understanding and treatment of substance use disorder and other mental illnesses marked by disruptions to decision making. 
bioRxiv preprint doi: https://doi.org/10.1101/2021.03.20.436233; this version posted June 9, 2021. The copyright holder for this preprint (which was not certified by peer review) is the author/funder, who has granted bioRxiv a license to display the preprint in perpetuity. It is made available under aCC-BY-NC-ND 4.0 International license.

Sias et al. 16

\section{MATERIALS AND METHODS}

\begin{tabular}{|c|c|c|c|c|}
\hline \multicolumn{5}{|c|}{ Key Resources Table } \\
\hline $\begin{array}{l}\text { Reagent type } \\
\text { (species) or } \\
\text { resource }\end{array}$ & Designation & $\begin{array}{l}\text { Source or } \\
\text { reference }\end{array}$ & Identifiers & $\begin{array}{l}\text { Additional } \\
\text { information }\end{array}$ \\
\hline $\begin{array}{l}\text { Recombinant DNA } \\
\text { reagent }\end{array}$ & $\begin{array}{l}\text { pENN.AAV5.CAMKII.GC } \\
\text { aMP6f.WPRE.SV40 }\end{array}$ & Addgene & $\begin{array}{l}\text { Cat: 100834-AAV5 } \\
\text { RRID: Addgene_100834 }\end{array}$ & Lot \# v59618 \\
\hline $\begin{array}{l}\text { Recombinant DNA } \\
\text { reagent }\end{array}$ & $\begin{array}{l}\text { rAAV5-CAMKIla- } \\
\text { eArchT3.0-eYFP }\end{array}$ & UNC-CH vector core & Deisseroth & Lot \# V4883D \\
\hline $\begin{array}{l}\text { Recombinant DNA } \\
\text { reagent }\end{array}$ & rAAV5-CAMKIIa-eYFP & $\begin{array}{l}\text { UNC-CH vector } \\
\text { core }\end{array}$ & Deisseroth & Lot \# AV4808I \\
\hline $\begin{array}{l}\text { Recombinant DNA } \\
\text { reagent }\end{array}$ & $\begin{array}{l}\text { pAAV8-hSyn-hM4D(Gi)- } \\
\text { mCherry }\end{array}$ & Addgene & $\begin{array}{l}\text { Cat: 50475-AAV8 } \\
\text { RRID: Addgene_50475 }\end{array}$ & Lot \# v5483 \\
\hline $\begin{array}{l}\text { Recombinant DNA } \\
\text { reagent }\end{array}$ & pAAV8-hSyn-mCherry & Addgene & $\begin{array}{l}\text { Cat: 114472-AAV8 } \\
\text { RRID: Addgene_114472 }\end{array}$ & \\
\hline Other & Optical fiber (photometry) & Neurophotometrics & & $\begin{array}{l}\text { Diameter: } 200 \\
\mu \mathrm{m} ; \mathrm{NA}: 0.37 \text {; } \\
\text { Length: } 8-8.5 \\
\mathrm{~mm}\end{array}$ \\
\hline Other & $\begin{array}{l}\text { Optical fiber } \\
\text { (manipulation) }\end{array}$ & Thorlabs & Cat: FT200UMT & $\begin{array}{l}\text { Core: } 200 \mu \mathrm{m} \text {; } \\
\text { NA: } 0.39 ; \\
\text { Length: } 8-8.5 \\
\mathrm{~mm}\end{array}$ \\
\hline Other & Optical ferrules & Kientec & Cat: FAZI-LC-230 & \\
\hline Other & Guide cannula & Plastics One & Cat: C313G/SPC & $\begin{array}{l}\text { Length: cut to } \\
4 \mathrm{~mm} \text { below } \\
\text { pedestal }\end{array}$ \\
\hline $\begin{array}{l}\text { Chemical } \\
\text { compound, drug }\end{array}$ & Clozapine $N$-oxide & Tocris & $\begin{array}{l}\text { Cat: } 4936 / 10 \\
\text { CAS: } 34233-69-7\end{array}$ & \\
\hline Other & $\begin{array}{l}\text { Dustless precision } \\
\text { Chocolate-flavored } \\
\text { purified pellets }\end{array}$ & Bio-Serv & Cat: F0299 & $45 \mathrm{mg}$ \\
\hline Other & Sucrose & Ralphs & UPC: 0001111083805 & \\
\hline Antibody & $\begin{array}{l}\text { Chicken anti-GFP } \\
\text { polyclonal antibody }\end{array}$ & Abcam & Cat: ab13970 & $1: 1000$ \\
\hline
\end{tabular}




\begin{tabular}{|c|c|c|c|c|}
\hline Antibody & $\begin{array}{l}\text { Goat, anti-chicken IgG, } \\
\text { Alexa Fluor } 488 \\
\text { conjugate }\end{array}$ & Abcam & Cat: ab150169 & $1: 500$ \\
\hline Antibody & $\begin{array}{l}\text { Rabbit anti-DsRed } \\
\text { polyclonal antibody }\end{array}$ & Takara Bio & Cat: 632496 & $1: 1000$ \\
\hline Antibody & $\begin{array}{l}\text { Goat anti-rabbit lgG, } \\
\text { Alexa Fluor } 594 \\
\text { conjugate }\end{array}$ & Invitrogen & Cat: A-11012 & $1: 500$ \\
\hline Other & $\begin{array}{l}\text { ProLong }{ }^{\mathrm{TM}} \text { Gold Antifade } \\
\text { Mountant with DAPI }\end{array}$ & Invitrogen & Cat: P36931 & \\
\hline $\begin{array}{l}\text { Chemical } \\
\text { compound, drug }\end{array}$ & Paraformaldehyde & Sigma & Cat: P6148 & \\
\hline Software, algorithm & MED-PC IV & $\begin{array}{l}\text { Med Associates, } \\
\text { Inc }\end{array}$ & RRID:SCR_012156 & \\
\hline Software, algorithm & GraphPad Prism & $\begin{array}{l}\text { GraphPad } \\
\text { Software }\end{array}$ & RRID:SCR_002798 & Version: 8 \\
\hline Software, algorithm & MatLab & MathWorks & RRID:SCR_001622 & $\begin{array}{l}\text { Version: } \\
2019 a\end{array}$ \\
\hline Software, algorithm & SPSS & IBM & RRID: SCR_019096 & Version: 26 \\
\hline Software, algorithm & Bonsai & Bonsai & RRID: SCR_017218 & Version: 2.3 \\
\hline Software, algorithm & Minianalysis & Synaptosoft & RRID: SCR_002184 & Version 6 \\
\hline Software, algorithm & BZ-X Analyze software & Keyence & & \\
\hline Software, algorithm & Zeiss Zen Blue software & Zeiss & & \\
\hline Software, algorithm & Illustrator & Adobe & RRID:SCR_010279 & \\
\hline Software, algorithm & ImageJ & $\mathrm{NIH}$ & RRID: SCR_003070 & \\
\hline Software, algorithm & Excel & Microsoft & RRID: SCR_016137 & \\
\hline
\end{tabular}

\section{Subjects.}

Male, Long Evans rats aged 8-10 weeks at the start of the experiment (Charles River Laboratories, Wilmington, MA) were group housed $(2 /$ cage $)$ in a temperature $\left(68-79^{\circ} \mathrm{F}\right)$ and humidity $(30-70 \%)$ regulated vivarium prior to surgery and then subsequently housed individually to preserve implants. Rats were provided with water ad libitum in the home cage and were maintained on a food-restricted 12-14 g daily diet (Lab Diet, St. Louis, MO) to maintain $\sim 85-90 \%$ free-feeding body weight. Rats were handled for 3-5 days prior to the onset of each 
experiment. Separate groups of naïve rats were used for each experiment. Experiments were performed during the dark phase of a 12:12 hr reverse dark/light cycle (lights off at 7AM). All procedures were conducted in accordance with the NIH Guide for the Care and Use of Laboratory Animals and were approved by the UCLA Institutional Animal Care and Use Committee.

\section{Surgery.}

Standard surgical procedures, described previously (Lichtenberg et al., 2017; Malvaez et al., 2015; Malvaez et al., 2019), were used for all surgeries. Rats were anesthetized with isoflurane (4-5\% induction, 1-3\% maintenance) and a nonsteroidal anti-inflammatory agent was administered pre- and post-operatively to minimize pain and discomfort.

Fiber photometry recordings. Surgery occurred prior to onset of behavioral training. Rats $(N=11)$ were infused bilaterally with adeno-associated virus (AAV) expressing the genetically encoded calcium indicator GCaMP6f under control of the calcium/calmodulin-dependent protein kinase (CaMKII) promoter (pENN.AAV5.CAMKII.GCaMP6f.WPRE.SV40, Addgene, Watertown, MA) to drive expression preferentially in principal neurons. Virus $(0.5 \mu \mathrm{l})$ was infused a rate of $0.1 \mu \mathrm{l} / \mathrm{min}$ into the BLA [AP: $-2.7(N=5)$ or $-3.0(N=6)$; $\mathrm{ML}: \pm 5.0$; DV: $-8.6 \mathrm{~mm}$ from bregma] using a 28-gauge injector. Injectors were left in place for an additional 10 minutes to ensure adequate diffusion and to minimize off-target spread along the injector tract. Optical fibers (200 $\mu \mathrm{m}$ diameter, 0.37 numerical aperture (NA), Neurophotometrics, San Diego, CA) were implanted bilaterally $0.2 \mathrm{~mm}$ dorsal to the infusion site to allow subsequent imaging of GCaMP fluctuations in BLA neurons. These procedures were replicated in a separate group of subjects $(N=6)$ that served as unpaired $\mathrm{CS}_{\varnothing}$ control. Behavioral training commenced approximately 3-4 weeks after surgery to allow sufficient expression in BLA neurons.

Optogenetic inhibition of BLA. Prior to the onset of behavioral training, rats were randomly assigned to a viral group and were infused bilaterally with AAV encoding either the inhibitory opsin archaerhodopsin T (ArchT; $N=$ 9; rAAV5-CAMKIla-eArchT3.0-eYFP, University of North Carolina Vector Core, Chapel Hill, NC) or the enhanced yellow fluorescent protein control (eYFP; $N=10$; rAAV5-CAMKIla-eYFP, University of North Carolina Vector Core) under control of the CaMKII promoter. Virus $(0.5 \mu \mathrm{l})$ was infused at a rate of $0.1 \mu \mathrm{l} / \mathrm{min}$ into the BLA (AP: -2.8; ML: \pm 5.0 ; DV: $-8.6 \mathrm{~mm}$ from bregma) using a 28-gauge injector. Injectors were left in place for an additional 10 minutes. Optical fibers (200 $\mu \mathrm{m}$ core, 0.39 NA, Thorlabs, Newton, NJ) held in ceramic ferrules (Kientec Systems, Stuart, FL) were implanted bilaterally $0.6 \mathrm{~mm}$ dorsal to the injection site to allow subsequent light delivery to ArchT- or eYFP-expressing BLA neurons. Identical surgical procedures were used for a separate yoked inhibition control group $(N=7)$. A third group $(N=5)$ also received bilateral infusion of rAAV5-CAMKIlaeArchT3.0-eYFP into the BLA, without fiber implants, for subsequent ex vivo electrophysiological validation of optical inhibition of BLA neurons. Experiments commenced 3 weeks after surgery to allow sufficient expression in BLA neurons.

Optogenetic inhibition of IOFC $\rightarrow B L A$ projections. Prior to the onset of behavioral training, rats were randomly assigned to a viral group and were infused with AAV encoding either the inhibitory opsin ArchT $(N=8$; rAAV5CAMKIla-eArchT3.0-eYFP) or eYFP control ( $N=8$; rAAV5-CAMKIla-eYFP). Virus $(0.3 \mu \mathrm{l})$ was infused at a rate of $0.1 \mathrm{\mu l} / \mathrm{min}$ bilaterally into the IOFC (AP: +3.3 ; ML: \pm 2.5 ; DV: $-5.4 \mathrm{~mm}$ from bregma) using a 28-gauge injector tip. Injectors were left in place for an additional 10 minutes. Optical fibers ( $200 \mu \mathrm{m}$ core, $0.39 \mathrm{NA})$ held in ceramic ferrules were implanted bilaterally in the BLA (AP: -2.7 ; ML: \pm 5.0 ; DV: $-8.0 \mathrm{~mm}$ from bregma) to allow subsequent light delivery to ArchT- or eYFP-expressing axons and terminals in the BLA. A separate group $(N=4)$ also received bilateral infusion of rAAV5-CAMKIla-eArchT3.0-eYFP into the IOFC, without fiber implants, for subsequent ex vivo electrophysiological validation of optical inhibition of IOFC terminals in the BLA. Experiments began 7-8 weeks following surgery to allow axonal transport to the BLA. 
Multiplexed optogenetic inhibition IOFC $\rightarrow B L A$ projections and chemogenetic inhibition of BLA $\rightarrow$ IOFC projections for serial circuit disconnection. Prior to the onset of behavioral training, rats were randomly assigned to viral group. The disconnection group $(N=10)$ was infused with AAV encoding the inhibitory opsin ArchT (rAAV5CAMKIla-eArchT3.0-eYFP; $0.3 \mu \mathrm{l}$ ) bilaterally at a rate of $0.1 \mu \mathrm{l} / \mathrm{min}$ into the IOFC (AP: +3.3 ; ML: \pm 2.5 ; DV: -5.4 $\mathrm{mm}$ from bregma) using a 28-gauge injector tip. Injectors were left in place for an additional 10 minutes. An optical fiber (200 $\mathrm{mm}$ core, $0.39 \mathrm{NA}$ ) held in a ceramic ferrule was implanted unilaterally (hemisphere counterbalanced across subjects) in the BLA (AP: -2.7 ; ML: \pm 5.0 ; DV: $-7.7 \mathrm{~mm}$ from dura) to allow subsequent light delivery to both the ipsilateral and contralateral ArchT-expressing axons and terminals in the BLA of only one hemisphere. During the same surgery, in the hemisphere contralateral to optical fiber placement, a second AAV was infused unilaterally at a rate of $0.1 \mu \mathrm{l} / \mathrm{min}$ into the BLA (AP: -3.0 ; ML: \pm 5.1 ; DV: -8.6 from bregma) to drive expression of the inhibitory designer receptor human M4 muscarinic receptor (hM4Di; pAAV8-hSyn$\mathrm{hM} 4 \mathrm{D}(\mathrm{Gi})$-mCherry, Addgene; $0.5 \mu \mathrm{l})$. A 22-gauge stainless-steel guide cannula was implanted unilaterally above the IOFC (AP: +3.0; ML: \pm 3.2 : DV: -4.0 ) of the BLA-hM4Di hemisphere to target the hM4D(Gi)-expressing axonal terminals, which are predominantly ipsilateral. This allowed subsequent optical inhibition of IOFC terminals in the BLA of one hemisphere and chemogenetic inhibition of BLA terminals in the IOFC of the other hemisphere, thus disconnecting the putative IOFC $\rightarrow$ BLA $\rightarrow$ IOFC circuit. Surgical procedures were identical for the fluorophore-only control group $(N=8)$, except with AAVs encoding only eYFP (IOFC; rAAV5-CAMKIIa-eYFP) and mCherry (BLA; pAAV8-hSyn-mCherry). A separate ipsilateral control group received the same surgical procedures as the experimental contralateral ArchT/hM4Di group, but with BLA pAAV8-hSyn-hM4D(Gi)-mCherry and IOFC guide cannula placed in the same hemisphere as the BLA optical fiber. Experiments began 7-8 weeks following surgery to allow sufficient viral expression and axonal transport. Two subjects became ill before testing and, thus, were excluded from the experiment (Contralateral ArchT/hM4Di, $N=1$; Ipsilateral ArchT/hM4Di, $N=$ 1).

\section{Behavioral Procedures.}

Apparatus. Training took place in Med Associates conditioning chambers (East Fairfield, VT) housed within sound- and light-attenuating boxes, described previously (Collins et al., 2019; Malvaez et al., 2015; Malvaez et al., 2019). For optogenetic manipulations, the chambers were outfitted with an Intensity Division Fiberoptic Rotary Joint (Doric Lenses, Quebec, QC, Canada) connecting the output fiber optic patch cords to a laser (Dragon Lasers, ChangChun, JiLin, China) positioned outside of the chamber.

Each chamber contained 2 retractable levers that could be inserted to the left and right of a recessed fooddelivery port (magazine) in the front wall. A photobeam entry detector was positioned at the entry to the food port. Each chamber was equipped with a syringe pump to deliver $20 \%$ sucrose solution in $0.1 \mathrm{ml}$ increments through a stainless-steel tube into one well of the food port and a pellet dispenser to deliver 45-mg purified chocolate food pellets (Bio-Serv, Frenchtown, NJ) into another well. Both a tone and white noise generator were attached to individual speakers on the wall opposite the levers and food-delivery port. A 3-watt, 24-volt house light mounted on the top of the back wall opposite the food-delivery port provided illumination and a fan mounted to the outer chamber provided ventilation and external noise reduction. Behavioral procedures were similar to that we have described previously (Lichtenberg et al., 2017; Lichtenberg \& Wassum, 2016; Malvaez et al., 2015)

Magazine conditioning. Rats first received one day of training to learn where to receive the sucrose and food pellet rewards. This included two separate sessions, separated by approximately $1 \mathrm{hr}$, order counterbalanced, one with 30 non-contingent deliveries of sucrose (60 s intertrial interval, ITI) and one with 30 food pellet deliveries (60 s ITI).

Pavlovian conditioning. Rats then received 8 sessions of Pavlovian conditioning (1 session/day on consecutive days) to learn to associate each of two auditory conditional stimuli (CSs; $80-82 \mathrm{db}, 2$ min duration), tone (1.5 
$\mathrm{kHz}$ ) or white noise, with a specific food reward, sucrose $(20 \%, 0.1 \mathrm{ml} /$ delivery) or purified chocolate pellets (45 mg; Bio-Serv). CS-reward pairings were counterbalanced at the start of each experiment. For half the subjects, tone was paired with sucrose and noise with pellets, with the other half receiving the opposite arrangement. Each session consisted of 8 tone and 8 white noise presentations, with the exception of the fiber photometry experiments, in which rats received 4 of each CS/session to reduce session time and, thus, minimize the effects of photobleaching. During each 2 min CS the associated reward was delivered on a $30 \mathrm{~s}$ random-time schedule, resulting in an average of 4 stimulus-reward pairings per trial. For the fiber photometry experiments, there was a minimum $15 \mathrm{~s}$ probe period after CS onset before the first reward delivery to allow us to dissociate signal fluctuations due to CS onset from those due to reward delivery/retrieval. CSs were delivered pseudo-randomly with a variable $2-4 \mathrm{~min}$ ITI (mean $=3 \mathrm{~min}$ ).

Procedures were identical for the unpaired $\mathrm{CS}_{\varnothing}$ control fiber photometry experiment, except no rewards were delivered during Pavlovian training. Subjects in this experiment instead received rewards in their home cage several hours after the $\mathrm{CS}_{\varnothing}$ sessions. On the day following the last $\mathrm{CS}_{\varnothing}$ session, these subjects received one session with non-contingent, unpredicted deliveries of sucrose and food pellets, each delivered on a $30 \mathrm{~s}$ random-time schedule during 4, 2-min periods (variable 2-4 min ITI, mean = $3 \mathrm{~min}$ ), resulting in an average of 16 deliveries of each outcome.

Instrumental conditioning. Rats were then given 11 days, minimum, of instrumental conditioning. They received 2 separate training sessions per day, one with the left lever and one with the right lever, separated by at least 1 $\mathrm{hr}$. Each action was reinforced with a different outcome (e.g., left press-chocolate pellets / right press-sucrose solution; counterbalanced with respect to the Pavlovian contingencies). Each session terminated after 30 outcomes had been earned or $45 \mathrm{~min}$ had elapsed. Actions were continuously reinforced on the first day and then escalated ultimately to a random-ratio 20 schedule of reinforcement.

Outcome-selective Pavlovian-to-instrumental transfer test. Following Pavlovian and instrumental conditioning, rats received an outcome-selective Pavlovian-to-instrumental transfer (PIT) test. On the day prior to the PIT test, rats were given a single 30-min extinction session during which both levers were available but pressing was not reinforced to establish a low level of responding. During the PIT test, both levers were continuously present, but pressing was not reinforced. After 5 min of lever-pressing extinction, each 2-min CS was presented separately 4 times in pseudorandom order, separated by a fixed 4-min inter-trial interval. No rewards were delivered during CS presentation.

Data collection. Lever presses and/or discrete entries into the food-delivery port were recorded continuously for each session. For both Pavlovian training and PIT test sessions, the 2-min periods prior to each CS onset served as the baseline for comparison of CS-induced elevations in lever pressing and/or food-port entries.

\section{In vivo fiber photometry.}

Fiber photometry was used to image bulk calcium activity in BLA neurons throughout each Pavlovian conditioning session. We simultaneously imaged GCaMP6f and control fluorescence in the BLA using a commercial fiber photometry system (Neurophotometrics Ltd., San Diego, CA). Two light-emitting LEDs (470nm: $\mathrm{Ca}^{2+}$-dependent GCaMP fluorescence; 415nm: autofluorescence, motion artifact, $\mathrm{Ca}^{2+}$-independent GCaMP fluorescence) were reflected off dichroic mirrors and coupled via a patch cord (fiber core diameter, $200 \mu \mathrm{m}$; Doric Lenses) to the implanted optical fiber. The intensity of the light for excitation was adjusted to $\sim 80 \mu \mathrm{W}$ at the tip of the patch cord. Fluorescence emission was passed through a $535 \mathrm{~nm}$ bandpass filter and focused onto the complementary metal-oxide semiconductor (CMOS) camera sensor through a tube lens. Samples were collected at $20 \mathrm{~Hz}$, interleaved between the 415 and 470 excitation channels, using a custom Bonsai (Lopes et al., 2015) workflow. Time stamps of task events were collected simultaneously through an additional synchronized camera 
aimed at the Med Associates interface, which sent light pulses coincident with task events. Signals were saved using Bonsai software and exported to MATLAB (MathWorks, Natick, MA) for analysis. Recordings were collected unilaterally from the hemisphere with the strongest fluorescence signal in the 470 channel at the start of the experiment, which was kept consistent throughout the remainder of the experiment. Animals were habituated to the optical tether during the magazine conditioning sessions, but no light was delivered.

\section{Optogenetic inhibition of BLA neurons.}

Optogenetic inhibition was used to attenuate the activity of ArchT-expressing BLA neurons at the time of stimulus-outcome pairing during each CS during each Pavlovian conditioning session. Animals were habituated to the optical tether $(200 \mu \mathrm{m}, 0.22 \mathrm{NA}$, Doric) during the magazine conditioning sessions, but no light was delivered. During each Pavlovian conditioning session, green light $(532 \mathrm{~nm} ; 10 \mathrm{~mW})$ was delivered to the BLA via a laser (Dragon Lasers, ChangChun) connected through a ceramic mating sleeve (Thorlabs) to the ferrule implanted on the rat. Light was delivered continuously for 5 seconds concurrent with each reward delivery. If the reward was retrieved (first food-port entry after reward delivery) while the light was still being delivered (i.e., within $5 \mathrm{~s}$ of reward delivery), then the light delivery was extended to $5 \mathrm{~s}$ from the time of the retrieval. If the reward was retrieved after the laser had gone off, then the retrieval entry triggered an additional $5 \mathrm{~s}$ continuous illumination. To control for the overall amount of inhibition, a separate control group received green light during the 2-min preCS baseline periods with the same number, duration, and pattern as the experimental group. Light effects were estimated to be restricted to the BLA based on predicted irradiance values (https://web.stanford.edu/group/dlab/cgi-bin/graph/chart.php). Following Pavlovian conditioning, rats proceeded through instrumental conditioning and the PIT test, as above. Light was not delivered during these subsequent phases of the experiment.

\section{Optogenetic inhibition of IOFC $\rightarrow B L A$ projections.}

Optogenetic inhibition was used to attenuate the activity of ArchT-expressing IOFC $\rightarrow$ BLA terminals at the time of stimulus-outcome pairing during each CS during each Pavlovian conditioning session. Procedures were identical to those for BLA inhibition above. Green light $(532 \mathrm{~nm} ; 10 \mathrm{~mW})$ was delivered to the BLA continuously for 5 seconds concurrent with each reward delivery and/or retrieval during Pavlovian conditioning.

\section{Multiplexed optogenetic inhibition of IOFC $\rightarrow B L A$ projections during Pavlovian conditioning and chemogenetic inhibition of BLA $\rightarrow I O F C$ projections during the Pavlovian-to-instrumental transfer test for serial circuit disconnection.}

We multiplexed optogenetic inhibition of IOFC $\rightarrow$ BLA projection activity during stimulus-outcome pairing during Pavlovian conditioning with chemogenetic inhibition of BLA $\rightarrow$ IOFC projection activity during the PIT test to perform a serial circuit disconnection and ask whether activity in IOFC $\rightarrow$ BLA projections mediates the encoding of the stimulus-outcome memory that is later retrieved via activation of BLA $\rightarrow$ IOFC projections (Lichtenberg et al., 2017). That is, whether IOFC $\rightarrow$ BLA $\rightarrow$ IOFC is a functional circuit for the encoding (IOFC $\rightarrow B L A)$ and subsequent use for guiding decision making (BLA $\rightarrow$ IOFC) of appetitive, sensory-specific, stimulus-outcome memories. To achieve the serial circuit disconnection, in the experimental group, we optically inactivated ipsilateral and contralateral IOFC input to the BLA of only one hemisphere during stimulus-outcome pairing during Pavlovian conditioning, and then chemogenetically inactivated predominantly ipsilateral (Lichtenberg et al., 2017) BLA axons and terminals in the IOFC of the other hemisphere during the PIT test. This leaves one of each pathway undisrupted to mediate the stimulus-outcome learning (IOFC $\rightarrow B L A)$ and retrieval (BLA $\rightarrow \mid O F C)$, but if IOFC $\rightarrow$ BLA $\rightarrow$ IOFC forms a functional stimulus-outcome memory circuit, then we will have disconnected the circuit in each hemisphere. 
Optogenetic inhibition of IOFC $\rightarrow B L A$ projections during Pavlovian conditioning. Optogenetic inhibition was used to attenuate the activity of ArchT-expressing IOFC $\rightarrow$ BLA terminals of one hemisphere at the time of stimulusoutcome pairing (reward delivery and retrieval) during each CS during each Pavlovian conditioning session. Procedures were identical to those described above, except that green light $(532 \mathrm{~nm} ; 10 \mathrm{~mW})$ was delivered unilaterally to the BLA continuously for 5 seconds concurrent with each reward delivery and retrieval during Pavlovian conditioning.

Chemogenetic inhibition of BLA $\rightarrow$ IOFC projections during the Pavlovian-to-instrumental transfer test. Chemogenetic inhibition was used to inactivate hM4Di-expressing BLA axons and terminals in the IOFC of one hemisphere during the PIT test. For the contralateral ArchT/hM4Di group, chemogenetic inhibition occurred in the hemisphere opposite to the one that received optical inhibition of IOFC $\rightarrow$ BLA projections during learning, thus achieving the disconnection. In a separate ipsilateral control group, the chemogenetic inhibition occurred on the same side as optical inhibition of IOFC $\rightarrow$ BLA projections during learning, leaving the entire circuit undisrupted in one hemisphere, while controlling for unilateral inhibition of each pathway. We selected chemogenetic inhibition so it could be multiplexed with optogenetic inhibition and to allow inhibition throughout the duration of the PIT test. CNO (Tocris Bioscience, Sterling Heights, MI) was dissolved in aCSF to $1 \mathrm{mM}$ and $0.25 \mu \mathrm{L}$ was intracranially infused over $1 \mathrm{~min}$ into the IOFC as previously described (Lichtenberg et al., 2017). Injectors were left in place for at least 1 additional min to allow for drug diffusion. The PIT test commenced within 5-10 min following infusion. CNO dose was selected based on evidence of both its behavioral effectiveness and ability to attenuate the activity of hM4Di-expressing BLA terminals in the IOFC (Lichtenberg et al., 2017). We have also demonstrated that this dose of CNO when infused into the IOFC has no effect on reward-related behavior in the absence of the hM4Di transgene (Lichtenberg et al., 2017).

\section{Ex vivo electrophysiology.}

Whole-cell patch clamp recordings were used to validate the efficacy of optical inhibition of BLA principal neuron activity and IOFC terminal activity in the BLA. Recordings were performed in brain slices from $\sim 3-4$ month old rats 3-4 (BLA cell body inhibition) or 7-8 (IOFC $\rightarrow$ BLA inhibition) weeks following surgery. To prepare brain slices, rats were deeply anesthetized with isoflurane and perfused transcardially with an ice-cold, oxygenated NMDGbased slicing solution containing (in mM): 30 NaHCO3, 20 HEPES, 1.25 NaH2PO4, 102 NMDG, 40 glucose, 3 $\mathrm{KCl}, 0.5 \mathrm{CaCl} 2-2 \mathrm{H} 2 \mathrm{O}, 10 \mathrm{MgSO} 4-\mathrm{H} 2 \mathrm{O}$ (pH adjusted to 7.3-7.35, osmolality 300-310 mOsm/L). Brains were extracted and immediately placed in ice-cold, oxygenated NMDG slicing solution. Coronal slices (350 $\mu \mathrm{m})$ were cut using a vibrating microtome (VT1000S; Leica Microsystems, Germany), transferred to an incubating chamber containing oxygenated NMDG slicing solution warmed to $32-34{ }^{\circ} \mathrm{C}$, and allowed to recover for 15 min before being transferred to an artificial cerebral spinal fluid (aCSF) solution containing (in $\mathrm{mM}$ ): $130 \mathrm{NaCl}, 3 \mathrm{KCl}, 1.25$ $\mathrm{NaH} 2 \mathrm{PO} 4,26 \mathrm{NaHCO} 3,2 \mathrm{MgCl} 2,2 \mathrm{CaCl}$, and 10 glucose) oxygenated with 95\% O2, 5\% CO2 (pH 7.2-7.4, osmolality $290-310 \mathrm{mOsm} / \mathrm{L}, 32-34^{\circ} \mathrm{C}$ ). After $15 \mathrm{~min}$, slices were moved to room temperature and allowed to recover for $\sim 30$ additional min prior to recording. All recordings were performed using an upright microscope (Olympus BX51WI, Center Valley, PA) equipped with differential interference contrast optics and fluorescence imaging (QIACAM fast 1394 monochromatic camera with Q-Capture Pro software, QImaging, Surrey, BC, Canada). Patch pipettes (3-5 $\mathrm{M} \Omega$ resistance) contained a Cesium methanesulfonate-based internal recording solution (in mM): 125 Cs-methanesulfonate, 4 NaCl, 1 MgCl2, 5 MgATP, 9 EGTA, 8 HEPES, 1 GTP-Tris, 10 phosphocreatine, and 0.1 leupeptin; pH 7.2 with $\mathrm{CsOH}, 270-280 \mathrm{mOsm})$. Biocytin (0.2\%, Sigma-Aldrich, St. Louis, MO) was included in the internal recording solution for subsequent postsynaptic cell visualization and identification. Recordings were obtained using a MultiClamp 700B Amplifier (Molecular Devices, Sunnyvale, CA) and the PCLAMP 10.3 acquisition software. 
Validation of BLA principal neuron optogenetic inhibition. Whole-cell patch clamp recordings in current-clamp mode were obtained from BLA principal neurons expressing ArchT-eYFP ( $N=12$ cells, 5 subjects). Visible eYFPexpressing cell bodies were identified in the BLA for recordings. After breaking through the membrane, recordings were obtained from cells while injecting suprathreshold depolarizing current (1 s). Current injection intensities that resulted in 8-15 action potentials were selected for recordings (100-800 pA). Electrode access resistances were maintained at $<30 \mathrm{M} \Omega$. Green light $(535 \mathrm{~nm}, 1 \mathrm{~s}$ pulse, 0.25-1 mW; CoolLED Ltd, Andover, UK) was delivered through the epifluorescence illumination pathway using Chroma Technologies filter cubes to activate ArchT and inhibit BLA cell bodies. The number of action potentials recorded in ArchT-expressing cells injected with suprathreshold current were recorded both prior to and after green light illumination.

Validation of IOFC terminal optogenetic inhibition in the BLA. Whole-cell patch-clamp recordings were collected in voltage-clamp mode. Visible eYFP-expressing axons and terminals were identified in the BLA and recordings were obtained from postsynaptic BLA neurons located only in highly fluorescent regions. After breaking through the membrane, recordings were obtained while holding the membrane potential at $-70 \mathrm{mV}$. Electrode access resistances were maintained at $<30 \mathrm{M} \Omega$. Spontaneous excitatory postsynaptic currents (sEPSCs) were recorded in the presence of the $\mathrm{GABA}_{\mathrm{A}}$ receptor antagonist bicuculline $(10 \mu \mathrm{M})$. Fifteen seconds of baseline recordings of sEPSCs were obtained prior to exposure to green light. Following baseline measurements, recordings of sEPSCs were obtained during continuous exposure to green light $(535 \mathrm{~nm}, 0.5 \mathrm{~mW})$ for $15 \mathrm{~s}$. Spontaneous EPSC events were analyzed offline using the automatic detection protocol within the MiniAnalysis software (Synaptosoft, version 6.0), and then were checked manually blinded to light condition.

\section{Histology.}

Following the behavioral experiments, rats were deeply anesthetized with Nembutal and transcardially perfused with phosphate buffered saline (PBS) followed by $4 \%$ paraformaldehyde (PFA). Brains were removed and postfixed in 4\% PFA overnight, placed into $30 \%$ sucrose solution, then sectioned into $30-40 \mu \mathrm{m}$ slices using a cryostat and stored in PBS or cryoprotectant.

eYFP fluorescence was used to confirm ArchT expression in IOFC and BLA cell bodies. mCherry expression was used to confirm $\mathrm{hM} 4 \mathrm{D}(\mathrm{Gi})$ in BLA cell bodies. Immunofluorescence was used to confirm expression of ArchT-eYFP in IOFC axons and terminals in the BLA. Floating coronal sections were washed 3 times in 1x PBS for $30 \mathrm{~min}$ and then blocked for $1-1.5 \mathrm{hr}$ at room temperature in a solution of $3 \%$ normal goat serum and $0.3 \%$ Triton X-100 dissolved in PBS. Sections were then washed 3 times in PBS for 15 min and incubated in blocking solution containing chicken anti-GFP polyclonal antibody (1:1000; Abcam, Cambridge, MA) with gentle agitation at $4^{\circ} \mathrm{C}$ for 18-22 hr. Sections were next rinsed 3 times in PBS for 30 min and incubated with goat anti-chicken IgY, Alexa Fluor 488 conjugate (1:500; Abcam) at room temperature for $2 \mathrm{hr}$. Sections were washed a final 3 times in PBS for 30 min. Immunofluorescence was also used to confirm expression of hM4Di-mCherry in BLA axons and terminals in the IOFC. The signal for axonal expression of hM4D(Gi)-mCherry in terminals in the IOFC was immunohistochemically amplified following procedures described previously (Lichtenberg et al., 2017). Briefly, floating coronal sections were rinsed in PBS and blocked for 1-2 hr at room temperature in a solution of $10 \%$ normal goat serum and $0.5 \%$ Triton X-100 dissolved in PBS and then incubated in blocking solution containing rabbit anti-DsRed polyclonal antibody (1:1000; Takara Bio, Mountain View, CA) with gentle agitation at $4^{\circ} \mathrm{C}$ for 18-22 hr. Sections were next rinsed in blocking solution and incubated with goat anti-rabbit IgG, Alexa Fluor 594 conjugate (1:500; Invitrogen, Waltham, MA) for $2 \mathrm{hr}$. Slices were mounted on slides and coverslipped with ProLong Gold mounting medium with DAPI. Images were acquired using a Keyence BZ-X710 microscope (Keyence, El Segundo, CA) with a 4x,10x, and 20x objective (CFI Plan Apo), CCD camera, and BZ-X Analyze software or a Zeiss apotome confocal microscope (Zeiss, Oberkochen, Germany) and Zeiss Zen Blue software (Zeiss). Subjects with off-target viral, fiber, and/or cannula placements were removed from the dataset (Fiber 
photometry: $N=2$; Fiber photometry $\mathrm{CS}_{\varnothing}$ control $N=0$; BLA ArchT: $N=2$; BLA ArchT yoked control: $N=1$; Contralateral disconnection, $N=6$; Ipsilateral control $N=7$ ).

\section{Data analysis.}

Behavioral analysis. Behavioral data were processed with Microsoft Excel (Microsoft, Redmond, WA). Left and/or right lever presses and/or entries into the food-delivery port were collected continuously for each training and test session. Acquisition of the Pavlovian conditional food-port approach response was assessed by computing an elevation ratio of the rate of entries into the food-delivery port (entries/min) during the CS prior to reward delivery (CS-probe) relative to 2-min baseline periods immediately prior to CS onset [(CS probe entry rate)/(CS probe entry rate + preCS entry rate)]. Data were averaged across trials for each CS and then averaged across the two CSs. We also compared the rate of food-port entries between the CS probe and the preCS baseline periods (see Figures 1-1a, 3-2a, 4-2a, 5-2a). Press rates on the last day of instrumental training were averaged across levers and compared between groups to test for any differences in the acquisition of lever press responding during instrumental training. No significant group differences were detected in any of the experiments (see Figures 1-1b, 3-2b, 4-2b, 5-2b). For the PIT test, lever pressing during the 2-min baseline periods immediately prior to the onset of each CS was compared with that during the 2-min CS periods. For both the baseline and CS periods, lever pressing was separated for presses on the lever that, during training, earned the same outcome as the presented cue (i.e., preCS-Same and CS-Same presses) versus those on the other available lever (i.e., preCS-Different and CS-Different presses). To evaluate the influence of CS presentation on lever pressing, we computed an elevation ratio for each lever [(CS-Same presses)/(CS-Same presses + preCSSame presses)] and [(CS-Different presses)/(CS-Different presses + preCS-Different presses)]. In all cases, there were no significant differences in baseline presses between levers in the absence of the CSs (Lever: lowest $P=0.33, \mathrm{~F}_{1,14}=1.02$ ), and no effect of group on baseline lever pressing (Group: lowest $P=0.54, \mathrm{~F}_{2,23}=0.63$; Group $x$ Lever lowest $\left.P=0.21, \mathrm{~F}_{1,14}=1.71\right)$. To evaluate the influence of CS presentation on food-port entries, i.e., the conditional goal-approach responses, we also computed an elevation ratio [(CS entries)/(CS entries + preCS entries)]. Data were averaged across trials for each CS and then averaged across the two CSs. We also compared the rate of pressing on each lever and, separately, food-port entries between the CS and preCS baseline periods (see Figures 1-1c-d, 3-2c-d, 4-2c-d, 5-2c-d).

Fiber photometry data analysis. Data were pre-processed using a custom-written pipeline in MATLAB (MathWorks, Natick, MA). Data from the $415 \mathrm{~nm}$ isosbestic control channel were used to correct for motion artifacts and photobleaching. Using least-squares linear regression, the 415 signal was fit to the 470 signal. Change in fluorescence $(\Delta \mathrm{F} / \mathrm{F})$ at each time point was calculated by subtracting the fitted 415 signal from the 470 signal and normalizing to the fitted 415 data [(470-fitted 415)/fitted 415)] (See Figure 1-2). The $\Delta F / F$ data were then Z-scored $[(\Delta F / F$ - mean $\Delta F / F) / \operatorname{std}(\Delta F / F)]$. Using a custom MATLAB workflow, Z-scored traces were then aligned to CS onset, reward delivery, reward retrieval (first food-port entry after reward delivery), and foodport entries without reward present during the CS probe period (after CS before first reward delivery) during the CS for each trial. Peak magnitude and AUC were calculated on the Z-scored trace for each trial using 3-s preevent baseline and 3-s post-event windows. Data were averaged across trials and then across CSs. Session data were excluded if no transient calcium fluctuations were detected on the $470 \mathrm{~nm}$ channel above the isosbestic channel or if poor linear fit was detected due to excessive motion artifact. To examine the progression in BLA activity across training, we compared data across conditioning sessions $1,2,3 / 4,5 / 6$, and $7 / 8$. Thus, data from the mid and latter training sessions were averaged across bins of 2 training sessions. Subjects without reliable data from at least one session per bin were excluded (CS+N=5; $\mathrm{CS}_{\varnothing} N=1$ ). We were able to obtain reliable imaging data all of the 8 training sessions from $N=8$ of the 11 total final subjects that received CSreward pairing (see Figure 1-3). 
Ex vivo electrophysiology. The number of action potentials evoked by suprathreshold current injection was compared before and during exposure to green light to confirm the inhibitory effect of ArchT in BLA principal neurons. To assess the effect of ArchT activation in IOFC $\rightarrow$ BLA terminals, the frequency of sEPSCs was compared before and during green light exposure.

Statistical analysis. Datasets were analyzed by two-tailed, paired and unpaired Student's $t$ tests, one-, two-, or three-way repeated-measures analysis of variance (ANOVA), as appropriate (GraphPad Prism, GraphPad, San Diego, CA; SPSS, IBM, Chicago, IL). Post hoc tests were corrected for multiple comparisons using the Bonferroni method. All data were tested for normality prior to analysis with ANOVA and the Greenhouse-Geisser correction was applied to mitigate the influence of unequal variance between conditions. Alpha levels were set at $P<0.05$.

\section{Rigor and reproducibility.}

Group sizes were estimated a priori based on prior work using male Long Evans rats in this behavioral task (Lichtenberg et al., 2017; Lichtenberg \& Wassum, 2016; Malvaez et al., 2015) and to ensure counterbalancing of CS-reward and Lever-reward pairings. Investigators were not blinded to viral group because they were required to administer virus. All behaviors were scored using automated software (MedPC). Each primary experiment included at least 1 replication cohort and cohorts were balanced by viral group, CS-reward and Leverreward pairings, hemisphere etc. prior to the start of the experiment. 


\section{DATA AND CODE AVAILABILITY}

All data and code support the findings of this study are available from the corresponding author upon request and via the source data and code files associated with this manuscript.

\section{ACKNOWLEDGEMENTS}

We would like to thank Dr. Avishek Adhikari for assistance setting up fiber photometry. We would also like to acknowledge the very helpful feedback from Dr. Alicia Izquierdo, Dr. Melissa Sharpe, Dr. Melissa Malvaez, and Dr. Avishek Adhikari on this manuscript. Lastly, we would like to acknowledge the generous infrastructure support from the Staglin Center for Behavior and Brain Sciences.

\section{ADDITIONAL INFORMATION}

\section{Funding}

\begin{tabular}{|l|l|l|}
\hline Funder & Grant reference number & Author \\
\hline National Institute of Drug Abuse & DA035443 & Kate M. Wassum \\
\hline National Science Foundation & & Ana C. Sias \\
\hline
\end{tabular}

\section{Author contributions}

ACS, AKM, and KMW designed the research, analyzed, and interpreted the data. ACS conducted the fiber photometry and multiplexed optogenetic/chemogenetic inhibition experiments and, along with KMW, analyzed these data. AMW contributed to the design of the fiber photometry analysis pipeline. AKM conducted the BLA and IOFC $\rightarrow$ BLA inhibition experiments. ACS contributed to the completion and analysis of these data. SW, VYG, and CMG assisted with data collection. TMW, CMG, and SW assisted with surgery and histology. MSL, SMH, CC, and KMW designed, and SMH and CC conducted the electrophysiology validation and, along with ACS and KMW, analyzed the resultant data. ACS and KMW wrote the manuscript.

\section{Ethics}

Animal experimentation: All procedures were in accordance with the US National Institutes of Health (NIH) Guide for the Care and Use of Laboratory Animals and were approved by the UCLA Institutional Animal Care and Use Committee (protocol 2012-021).

Competing financial interests: The authors declare no biomedical financial interests or potential conflicts of interest. 
bioRxiv preprint doi: https://doi.org/10.1101/2021.03.20.436233; this version posted June 9, 2021. The copyright holder for this preprint (which was not certified by peer review) is the author/funder, who has granted bioRxiv a license to display the preprint in perpetuity. It is made available under aCC-BY-NC-ND 4.0 International license.

Sias et al. 27

\section{REFERENCES}

Aggleton, J. P., Burton, M. J., \& Passingham, R. E. (1980). Cortical and subcortical afferents to the amygdala of the rhesus monkey (Macaca mulatta). Brain Res, 190(2), 347-368. https://doi.org/10.1016/0006-8993(80)90279-6

Arguello, A. A., Richardson, B. D., Hall, J. L., Wang, R., Hodges, M. A., Mitchell, M. P., Stuber, G. D., Rossi, D. J., \& Fuchs, R. A. (2017). Role of a Lateral Orbital Frontal Cortex-Basolateral Amygdala Circuit in Cue-Induced Cocaine-Seeking Behavior. Neuropsychopharmacology, 42(3), 727-735. https://doi.org/10.1038/npp.2016.157

Balleine, B. W., \& Dickinson, A. (1998). Goal-directed instrumental action: contingency and incentive learning and their cortical substrates. Neuropharmacology, 37(4-5), 407-419.

Baltz, E. T., Yalcinbas, E. A., Renteria, R., \& Gremel, C. M. (2018). Orbital frontal cortex updates state-induced value change for decisionmaking. Elife, 7. https://doi.org/10.7554/eLife.35988

Barreiros, I. V., Panayi, M. C., \& Walton, M. E. (2021). Organization of Afferents along the Anterior-posterior and Medial-lateral Axes of the Rat Orbitofrontal Cortex. Neuroscience, 460, 53-68. https://doi.org/10.1016/j.neuroscience.2021.02.017

Baxter, M. G., \& Murray, E. A. (2002). The amygdala and reward. Nat Rev Neurosci, 3(7), 563-573. https://doi.org/10.1038/nrn875

Baxter, M. G., Parker, A., Lindner, C. C., Izquierdo, A. D., \& Murray, E. A. (2000). Control of response selection by reinforcer value requires interaction of amygdala and orbital prefrontal cortex. J Neurosci, 20(11), 4311-4319.

Belova, M. A., Paton, J. J., \& Salzman, C. D. (2008). Moment-to-moment tracking of state value in the amygdala. J Neurosci, 28(40), $10023-10030$.

Beyeler, A., Chang, C. J., Silvestre, M., Lévêque, C., Namburi, P., Wildes, C. P., \& Tye, K. M. (2018). Organization of Valence-Encoding and Projection-Defined Neurons in the Basolateral Amygdala. Cell Rep, 22(4), 905-918. https://doi.org/10.1016/j.celrep.2017.12.097

Beyeler, A., Namburi, P., Glober, G. F., Simonnet, C., Calhoon, G. G., Conyers, G. F., Luck, R., Wildes, C. P., \& Tye, K. M. (2016). Divergent Routing of Positive and Negative Information from the Amygdala during Memory Retrieval. Neuron, 90(2), 348-361. https://doi.org/10.1016/j.neuron.2016.03.004

Blundell, P., Hall, G., \& Killcross, S. (2001). Lesions of the basolateral amygdala disrupt selective aspects of reinforcer representation in rats. J Neurosci, 21(22), 9018-9026.

Bordi, F., \& LeDoux, J. (1992). Sensory tuning beyond the sensory system: an initial analysis of auditory response properties of neurons in the lateral amygdaloid nucleus and overlying areas of the striatum. J Neurosci, 12(7), 2493-2503.

Bordi, F., LeDoux, J., Clugnet, M. C., \& Pavlides, C. (1993). Single-unit activity in the lateral nucleus of the amygdala and overlying areas of the striatum in freely behaving rats: rates, discharge patterns, and responses to acoustic stimuli. Behav Neurosci, 107(5), 757-769. https://doi.org/10.1037/0735-7044.107.5.757

Bradfield, L. A., \& Hart, G. (2020). Rodent medial and lateral orbitofrontal cortices represent unique components of cognitive maps of task space. Neurosci Biobehav Rev, 108, 287-294. https://doi.org/10.1016/j.neubiorev.2019.11.009

Butler, R. K., Sharko, A. C., Oliver, E. M., Brito-Vargas, P., Kaigler, K. F., Fadel, J. R., \& Wilson, M. A. (2011). Activation of phenotypicallydistinct neuronal subpopulations of the rat amygdala following exposure to predator odor. Neuroscience, 175, $133-144$. https://doi.org/10.1016/j.neuroscience.2010.12.001

Carmichael, S. T., \& Price, J. L. (1995). Limbic connections of the orbital and medial prefrontal cortex in macaque monkeys. J Comp Neurol, 363(4), 615-641. https://doi.org/10.1002/cne.903630408

Chen, T. W., Wardill, T. J., Sun, Y., Pulver, S. R., Renninger, S. L., Baohan, A., Schreiter, E. R., Kerr, R. A., Orger, M. B., Jayaraman, V., Looger, L. L., Svoboda, K., \& Kim, D. S. (2013). Ultrasensitive fluorescent proteins for imaging neuronal activity. Nature, 499(7458), 295-300. https://doi.org/10.1038/nature12354

Collins, A. L., Aitken, T. J., Huang, I. W., Shieh, C., Greenfield, V. Y., Monbouquette, H. G., Ostlund, S. B., \& Wassum, K. M. (2019). Nucleus Accumbens Cholinergic Interneurons Oppose Cue-Motivated Behavior. Biol Psychiatry. https://doi.org/10.1016/j.biopsych.2019.02.014

Colwill, R. M., \& Motzkin, D. K. (1994). Encoding of the unconditioned stimulus in Pavlovian conditioning. Animal Learning \& Behavior, 22(4), 384-394.

Constantinople, C. M., Piet, A. T., Bibawi, P., Akrami, A., Kopec, C., \& Brody, C. D. (2019). Lateral orbitofrontal cortex promotes trial-bytrial learning of risky, but not spatial, biases. Elife, 8. https://doi.org/10.7554/eLife.49744

Corbit, L. H., \& Balleine, B. W. (2005). Double dissociation of basolateral and central amygdala lesions on the general and outcomespecific forms of pavlovian-instrumental transfer. J Neurosci, 25(4), 962-970.

Corbit, L. H., \& Balleine, B. W. (2016). Learning and Motivational Processes Contributing to Pavlovian-Instrumental Transfer and Their Neural Bases: Dopamine and Beyond. Curr Top Behav Neurosci. https://doi.org/10.1007/7854 2015388

Corbit, L. H., Leung, B. K., \& Balleine, B. W. (2013). The role of the amygdala-striatal pathway in the acquisition and performance of goaldirected instrumental actions. J Neurosci, 33(45), 17682-17690. https://doi.org/10.1523/JNEUROSCI.3271-13.2013

Costa, V. D., Dal Monte, O., Lucas, D. R., Murray, E. A., \& Averbeck, B. B. (2016). Amygdala and Ventral Striatum Make Distinct Contributions to Reinforcement Learning. Neuron, 92(2), 505-517. https://doi.org/10.1016/j.neuron.2016.09.025

Costa, V. D., Mitz, A. R., \& Averbeck, B. B. (2019). Subcortical Substrates of Explore-Exploit Decisions in Primates. Neuron, 103(3), 533545.e535. https://doi.org/10.1016/j.neuron.2019.05.017

Cromwell, H. C., Anstrom, K., Azarov, A., \& Woodward, D. J. (2005). Auditory inhibitory gating in the amygdala: single-unit analysis in the behaving rat. Brain Res, 1043(1-2), 12-23. https://doi.org/10.1016/j.brainres.2005.01.106

Crouse, R. B., Kim, K., Batchelor, H. M., Girardi, E. M., Kamaletdinova, R., Chan, J., Rajebhosale, P., Pittenger, S. T., Role, L. W., Talmage, D. A., Jing, M., Li, Y., Gao, X. B., Mineur, Y. S., \& Picciotto, M. R. (2020). Acetylcholine is released in the basolateral amygdala in response to predictors of reward and enhances the learning of cue-reward contingency. Elife, 9. https://doi.org/10.7554/eLife. 57335 
bioRxiv preprint doi: https://doi.org/10.1101/2021.03.20.436233; this version posted June 9, 2021. The copyright holder for this preprint (which was not certified by peer review) is the author/funder, who has granted bioRxiv a license to display the preprint in perpetuity. It is made available under aCC-BY-NC-ND 4.0 International license.

Sias et al. 28

Delamater, A. R. (2007). The role of the orbitofrontal cortex in sensory-specific encoding of associations in pavlovian and instrumental conditioning. Ann N Y Acad Sci, 1121, 152-173. https://doi.org/10.1196/annals.1401.030

Delamater, A. R. (2012). On the nature of CS and US representations in Pavlovian learning. Learn Behav, 40(1), 1-23. https://doi.org/10.3758/s13420-011-0036-4

Delamater, A. R., \& Oakeshott, S. (2007). Learning about multiple attributes of reward in Pavlovian conditioning. Ann N Y Acad Sci, 1104, 1-20. https://doi.org/10.1196/annals.1390.008

Derman, R. C., Bass, C. E., \& Ferrario, C. R. (2020). Effects of hM4Di activation in CamKII basolateral amygdala neurons and CNO treatment on sensory-specific vs. general PIT: refining PIT circuits and considerations for using CNO. Psychopharmacology (Berl), 237(5), 1249-1266. https://doi.org/10.1007/s00213-020-05453-8

Everitt, B. J., Cardinal, R. N., Hall, J., Parkinson, J. A., \& Robbins, T. W. (2000). Differential involvement of amygdala subsystems in appetitive conditioning and drug addiction. In The amygdala: A functional analysi (pp. 353-390).

Fanselow, M. S., \& Wassum, K. M. (2015). The Origins and Organization of Vertebrate Pavlovian Conditioning. Cold Spring Harb Perspect Biol. https://doi.org/10.1101/cshperspect.a021717

Fisher, S. D., Ferguson, L. A., Bertran-Gonzalez, J., \& Balleine, B. W. (2020). Amygdala-Cortical Control of Striatal Plasticity Drives the Acquisition of Goal-Directed Action. Curr Biol. https://doi.org/10.1016/j.cub.2020.08.090

Fiuzat, E. C., Rhodes, S. E., \& Murray, E. A. (2017). The role of orbitofrontal-amygdala interactions in updating action-outcome valuations in macaques. J Neurosci. https://doi.org/10.1523/JNEUROSCI.1839-16.2017

Fontanini, A., Grossman, S. E., Figueroa, J. A., \& Katz, D. B. (2009). Distinct subtypes of basolateral amygdala taste neurons reflect palatability and reward. $J$ Neurosci, 29(8), 2486-2495.

Gardner, M., \& Schoenbaum, G. (2020). The orbitofrontal cartographer. PsyArXiv. https://doi.org/10.31234/osf.io/4mrxy

Gilroy, K. E., Everett, E. M., \& Delamater, A. R. (2014). Response-Outcome versus Outcome-Response Associations in Pavlovian-toInstrumental Transfer: Effects of Instrumental Training Context. Int J Comp Psychol, 27(4), 585-597.

Goldstein, R. Z., \& Volkow, N. D. (2011). Dysfunction of the prefrontal cortex in addiction: neuroimaging findings and clinical implications. Nat Rev Neurosci, 12(11), 652-669. https://doi.org/10.1038/nrn3119

Groman, S. M., Keistler, C., Keip, A. J., Hammarlund, E., DiLeone, R. J., Pittenger, C., Lee, D., \& Taylor, J. R. (2019). Orbitofrontal Circuits Control Multiple Reinforcement-Learning Processes. Neuron. https://doi.org/10.1016/j.neuron.2019.05.042

Hatfield, T., Han, J. S., Conley, M., Gallagher, M., \& Holland, P. (1996). Neurotoxic lesions of basolateral, but not central, amygdala interfere with Pavlovian second-order conditioning and reinforcer devaluation effects. J Neurosci, 16(16), 5256-5265.

Heilbronner, S. R., Rodriguez-Romaguera, J., Quirk, G. J., Groenewegen, H. J., \& Haber, S. N. (2016). Circuit-Based Corticostriatal Homologies Between Rat and Primate. Biol Psychiatry, 80(7), 509-521. https://doi.org/10.1016/j.biopsych.2016.05.012

Howard, J. D., Gottfried, J. A., Tobler, P. N., \& Kahnt, T. (2015). Identity-specific coding of future rewards in the human orbitofrontal cortex. Proc Natl Acad Sci U S A, 112(16), 5195-5200. https://doi.org/10.1073/pnas.1503550112

Howard, J. D., \& Kahnt, T. (2018). Identity prediction errors in the human midbrain update reward-identity expectations in the orbitofrontal cortex. Nat Commun, 9(1), 1611. https://doi.org/10.1038/s41467-018-04055-5

Izquierdo, A., Darling, C., Manos, N., Pozos, H., Kim, C., Ostrander, S., Cazares, V., Stepp, H., \& Rudebeck, P. H. (2013). Basolateral amygdala lesions facilitate reward choices after negative feedback in rats. $J$ Neurosci, 33(9), 4105-4109. https://doi.org/10.1523/JNEUROSCI.4942-12.2013

Izquierdo, A., Suda, R. K., \& Murray, E. A. (2004). Bilateral orbital prefrontal cortex lesions in rhesus monkeys disrupt choices guided by both reward value and reward contingency. J Neurosci, 24(34), 7540-7548. https://doi.org/10.1523/JNEUROSCI.1921-04.2004

Janak, P. H., \& Tye, K. M. (2015). From circuits to behaviour in the amygdala. Nature, 517(7534), $284-292$. https://doi.org/10.1038/nature14188

Johansen, J. P., Tarpley, J. W., LeDoux, J. E., \& Blair, H. T. (2010). Neural substrates for expectation-modulated fear learning in the amygdala and periaqueductal gray. Nat Neurosci, 13(8), 979-986. https://doi.org/10.1038/nn.2594

Johnson, A. W., Gallagher, M., \& Holland, P. C. (2009). The basolateral amygdala is critical to the expression of pavlovian and instrumental outcome-specific reinforcer devaluation effects. J Neurosci, 29(3), 696-704.

Keiflin, R., Reese, R. M., Woods, C. A., \& Janak, P. H. (2013). The orbitofrontal cortex as part of a hierarchical neural system mediating choice between two good options. J Neurosci, 33(40), 15989-15998. https://doi.org/10.1523/JNEUROSCI.0026-13.2013

Klein-Flügge, M. C., Barron, H. C., Brodersen, K. H., Dolan, R. J., \& Behrens, T. E. (2013). Segregated encoding of reward-identity and stimulus-reward associations in human orbitofrontal cortex. J Neurosci, 33(7), 3202-3211. https://doi.org/10.1523/JNEUROSCI.2532-12.2013

Kochli, D. E., Keefer, S. E., Gyawali, U., \& Calu, D. J. (2020). Basolateral Amygdala to Nucleus Accumbens Communication Differentially Mediates Devaluation Sensitivity of Sign- and Goal-Tracking Rats. Front Behav Neurosci, 14,593645 https://doi.org/10.3389/fnbeh.2020.593645

Kruse, H., Overmier, J., Konz, W., \& Rokke, E. (1983). Pavlovian conditioned stimulus effects upon instrumental choice behavior are reinforcer specific. Learn Motiv, 14, 165-181.

Levin, J. R., Serlin, R.C., Seaman, \& M.A. (1994). A controlled powerful multiple-comparison strategy for several situations Psychological Bulletin, 115, 153-159.

Lichtenberg, N. T., Pennington, Z. T., Holley, S. M., Greenfield, V. Y., Cepeda, C., Levine, M. S., \& Wassum, K. M. (2017). Basolateral amygdala to orbitofrontal cortex projections enable cue-triggered reward expectations. $J$ Neurosci. https://doi.org/10.1523/JNEUROSCl.0486-17.2017

Lichtenberg, N. T., \& Wassum, K. M. (2016). Amygdala mu-opioid receptors mediate the motivating influence of cue-triggered reward expectations. Eur J Neurosci. https://doi.org/10.1111/ejn.13477

Liu, H., Tang, Y., Womer, F., Fan, G., Lu, T., Driesen, N., Ren, L., Wang, Y., He, Y., Blumberg, H. P., Xu, K., \& Wang, F. (2014). Differentiating patterns of amygdala-frontal functional connectivity in schizophrenia and bipolar disorder. Schizophr Bull, 40(2), 469-477. https://doi.org/10.1093/schbul/sbt044 
bioRxiv preprint doi: https://doi.org/10.1101/2021.03.20.436233; this version posted June 9, 2021. The copyright holder for this preprint (which was not certified by peer review) is the author/funder, who has granted bioRxiv a license to display the preprint in perpetuity. It is made available under aCC-BY-NC-ND 4.0 International license.

Sias et al. 29

Liu, J., Lyu, C., Li, M., Liu, T., Song, S., \& Tsien, J. Z. (2018). Neural Coding of Appetitive Food Experiences in the Amygdala. Neurobiol Learn Mem, 155, 261-275. https://doi.org/10.1016/j.nlm.2018.08.012

Lopatina, N., McDannald, M. A., Styer, C. V., Sadacca, B. F., Cheer, J. F., \& Schoenbaum, G. (2015). Lateral orbitofrontal neurons acquire responses to upshifted, downshifted, or blocked cues during unblocking. Elife, 4 , e11299. https://doi.org/10.7554/eLife.11299

Lopes, G., Bonacchi, N., Frazão, J., Neto, J. P., Atallah, B. V., Soares, S., Moreira, L., Matias, S., Itskov, P. M., Correia, P. A., Medina, R. E., Calcaterra, L., Dreosti, E., Paton, J. J., \& Kampff, A. R. (2015). Bonsai: an event-based framework for processing and controlling data streams. Front Neuroinform, 9, 7. https://doi.org/10.3389/fninf.2015.00007

Lucantonio, F., Gardner, M. P., Mirenzi, A., Newman, L. E., Takahashi, Y. K., \& Schoenbaum, G. (2015). Neural Estimates of Imagined Outcomes in Basolateral Amygdala Depend on Orbitofrontal Cortex. J Neurosci, 35(50), 16521-16530. https://doi.org/10.1523/JNEUROSCl.3126-15.2015

Lutas, A., Kucukdereli, H., Alturkistani, O., Carty, C., Sugden, A. U., Fernando, K., Diaz, V., Flores-Maldonado, V., \& Andermann, M. L. (2019). State-specific gating of salient cues by midbrain dopaminergic input to basal amygdala. Nat Neurosci, 22(11), 18201833. https://doi.org/10.1038/s41593-019-0506-0

Machado, C. J., \& Bachevalier, J. (2007). The effects of selective amygdala, orbital frontal cortex or hippocampal formation lesions on reward assessment in nonhuman primates. Eur J Neurosci, 25(9), 2885-2904. https://doi.org/10.1111/j.1460-9568.2007.05525.x

Malvaez, M., Greenfield, V. Y., Wang, A. S., Yorita, A. M., Feng, L., Linker, K. E., Monbouquette, H. G., \& Wassum, K. M. (2015). Basolateral amygdala rapid glutamate release encodes an outcome-specific representation vital for reward-predictive cues to selectively invigorate reward-seeking actions. Sci Rep, 5, 12511. https://doi.org/10.1038/srep12511

Malvaez, M., Shieh, C., Murphy, M. D., Greenfield, V. Y., \& Wassum, K. M. (2019). Distinct cortical-amygdala projections drive reward value encoding and retrieval. Nat Neurosci, 22(5), 762-769. https://doi.org/10.1038/s41593-019-0374-7

McDannald, M. A., Esber, G. R., Wegener, M. A., Wied, H. M., Liu, T. L., Stalnaker, T. A., Jones, J. L., Trageser, J., \& Schoenbaum, G. (2014). Orbitofrontal neurons acquire responses to 'valueless' Pavlovian cues during unblocking. Elife, 3, e02653. https://doi.org/10.7554/eLife.02653

Miller, K. J., Botvinick, M. M., \& Brody, C. D. (2018). Value Representations in Orbitofrontal Cortex Drive Learning, not Choice. bioRxiv, 245720. https://doi.org/10.1101/245720

Morecraft, R. J., Geula, C., \& Mesulam, M. M. (1992). Cytoarchitecture and neural afferents of orbitofrontal cortex in the brain of the monkey. J Comp Neurol, 323(3), 341-358. https://doi.org/10.1002/cne.903230304

Morse, A. K., Leung, B. K., Heath, E., Bertran-Gonzalez, J., Pepin, E., Chieng, B. C., Balleine, B. W., \& Laurent, V. (2020). Basolateral Amygdala Drives a GPCR-Mediated Striatal Memory Necessary for Predictive Learning to Influence Choice. Neuron, 106(5), 855-869.e858. https://doi.org/10.1016/j.neuron.2020.03.007

Muller, J., Corodimas, K. P., Fridel, Z., \& LeDoux, J. E. (1997). Functional inactivation of the lateral and basal nuclei of the amygdala by muscimol infusion prevents fear conditioning to an explicit conditioned stimulus and to contextual stimuli. Behav Neurosci, 111(4), 683-691. https://doi.org/10.1037//0735-7044.111.4.683

Muramoto, K., Ono, T., Nishijo, H., \& Fukuda, M. (1993). Rat amygdaloid neuron responses during auditory discrimination. Neuroscience, 52(3), 621-636.

Murray, E. A., \& Izquierdo, A. (2007). Orbitofrontal cortex and amygdala contributions to affect and action in primates. Ann N Y Acad Sci, 1121, 273-296. https://doi.org/10.1196/annals.1401.021

Málková, L., Gaffan, D., \& Murray, E. A. (1997). Excitotoxic lesions of the amygdala fail to produce impairment in visual learning for auditory secondary reinforcement but interfere with reinforcer devaluation effects in rhesus monkeys. $J$ Neurosci, $17(15), 6011-$ 6020.

Orsini, C. A., Hernandez, C. M., Singhal, S., Kelly, K. B., Frazier, C. J., Bizon, J. L., \& Setlow, B. (2017). Optogenetic Inhibition Reveals Distinct Roles for Basolateral Amygdala Activity at Discrete Time Points during Risky Decision Making. J Neurosci, 37(48), 11537-11548. https://doi.org/10.1523/JNEUROSCI.2344-17.2017

Ostlund, S. B., \& Balleine, B. W. (2007a). Orbitofrontal cortex mediates outcome encoding in Pavlovian but not instrumental conditioning. $J$ Neurosci, 27(18), 4819-4825.

Ostlund, S. B., \& Balleine, B. W. (2007b). The contribution of orbitofrontal cortex to action selection. Ann N Y Acad Sci, 1121, 174-192.

Ostlund, S. B., \& Balleine, B. W. (2008). Differential involvement of the basolateral amygdala and mediodorsal thalamus in instrumental action selection. J Neurosci, 28(17), 4398-4405.

Parkes, S. L., \& Balleine, B. W. (2013). Incentive Memory: Evidence the Basolateral Amygdala Encodes and the Insular Cortex Retrieves Outcome Values to Guide Choice between Goal-Directed Actions. J Neurosci, 33(20), 8753-8763. https://doi.org/10.1523/JNEUROSCI.5071-12.2013

Parkinson, J. A., Robbins, T. W., \& Everitt, B. J. (2000). Dissociable roles of the central and basolateral amygdala in appetitive emotional learning. Eur J Neurosci, 12(1), 405-413.

Passamonti, L., Fairchild, G., Fornito, A., Goodyer, I. M., Nimmo-Smith, I., Hagan, C. C., \& Calder, A. J. (2012). Abnormal anatomical connectivity between the amygdala and orbitofrontal cortex in conduct disorder. PLoS One, 7(11), e48789. https://doi.org/10.1371/journal.pone.0048789

Paton, J. J., Belova, M. A., Morrison, S. E., \& Salzman, C. D. (2006). The primate amygdala represents the positive and negative value of visual stimuli during learning. Nature, 439(7078), 865-870.

Paxinos, G., \& Watson, C. (1998). The rat brain in stereotaxic coordinates (4th ed.). Academic Press.

Pickens, C. L., Saddoris, M. P., Gallagher, M., \& Holland, P. C. (2005). Orbitofrontal lesions impair use of cue-outcome associations in a devaluation task. Behav Neurosci, 119(1), 317-322. https://doi.org/2005-01705-030 [pii]10.1037/0735-7044.119.1.317

Pickens, C. L., Saddoris, M. P., Setlow, B., Gallagher, M., Holland, P. C., \& Schoenbaum, G. (2003). Different roles for orbitofrontal cortex and basolateral amygdala in a reinforcer devaluation task. J Neurosci, 23(35), 11078-11084. 
bioRxiv preprint doi: https://doi.org/10.1101/2021.03.20.436233; this version posted June 9, 2021. The copyright holder for this preprint (which was not certified by peer review) is the author/funder, who has granted bioRxiv a license to display the preprint in perpetuity. It is made available under aCC-BY-NC-ND 4.0 International license.

Sias et al. 30

Pignatelli, M., \& Beyeler, A. (2019). Valence coding in amygdala circuits. Curr Opin Behav Sci, 26, 97-106. https://doi.org/10.1016/j.cobeha.2018.10.010

Price, J. L. (2007). Definition of the orbital cortex in relation to specific connections with limbic and visceral structures and other cortical regions. Ann N Y Acad Sci, 1121, 54-71. https://doi.org/10.1196/annals.1401.008

Pritchard, T. C., Edwards, E. M., Smith, C. A., Hilgert, K. G., Gavlick, A. M., Maryniak, T. D., Schwartz, G. J., \& Scott, T. R. (2005). Gustatory neural responses in the medial orbitofrontal cortex of the old world monkey. J Neurosci, 25(26), 6047-6056. https://doi.org/10.1523/JNEUROSCI.0430-05.2005

Ressler, K. J., \& Mayberg, H. S. (2007). Targeting abnormal neural circuits in mood and anxiety disorders: from the laboratory to the clinic. Nat Neurosci, 10(9), 1116-1124. https://doi.org/10.1038/nn1944

Rhodes, S. E., \& Murray, E. A. (2013). Differential effects of amygdala, orbital prefrontal cortex, and prelimbic cortex lesions on goaldirected behavior in rhesus macaques. J Neurosci, 33(8), 3380-3389. https://doi.org/10.1523/JNEUROSCI.4374-12.2013

Rich, E. L., \& Wallis, J. D. (2016). Decoding subjective decisions from orbitofrontal cortex. Nat Neurosci, 19(7), 973-980. https://doi.org/10.1038/nn.4320

Roesch, M. R., Calu, D. J., Esber, G. R., \& Schoenbaum, G. (2010). Neural correlates of variations in event processing during learning in basolateral amygdala. J Neurosci, 30(7), 2464-2471. https://doi.org/30/7/2464 [pii]10.1523/JNEUROSCI.5781-09.2010

Romanski, L. M., Clugnet, M. C., Bordi, F., \& LeDoux, J. E. (1993). Somatosensory and auditory convergence in the lateral nucleus of the amygdala. Behav Neurosci, 107(3), 444-450.

Rudebeck, P., \& Rich, E. (2018). Orbitofrontal cortex. Current Biology, 28(18), R1083-R1088.

Rudebeck, P. H., Mitz, A. R., Chacko, R. V., \& Murray, E. A. (2013). Effects of amygdala lesions on reward-value coding in orbital and medial prefrontal cortex. Neuron, 80(6), 1519-1531. https://doi.org/10.1016/j.neuron.2013.09.036

Rudebeck, P. H., \& Murray, E. A. (2014). The orbitofrontal oracle: cortical mechanisms for the prediction and evaluation of specific behavioral outcomes. Neuron, 84(6), 1143-1156. https://doi.org/10.1016/j.neuron.2014.10.049

Rudebeck, P. H., Ripple, J. A., Mitz, A. R., Averbeck, B. B., \& Murray, E. A. (2017). Amygdala contributions to stimulus-reward encoding in the macaque medial and orbital frontal cortex during learning. J Neurosci. https://doi.org/10.1523/JNEUROSCl.0933-16.2017

Saddoris, M. P., Gallagher, M., \& Schoenbaum, G. (2005). Rapid associative encoding in basolateral amygdala depends on connections with orbitofrontal cortex. Neuron, 46(2), 321-331. https://doi.org/S0896-6273(05)00160-1 [pii]10.1016/j.neuron.2005.02.018

Scarlet, J., Delamater, A. R., Campese, V., Fein, M., \& Wheeler, D. S. (2012). Differential involvement of the basolateral amygdala and orbitofrontal cortex in the formation of sensory-specific associations in conditioned flavor preference and magazine approach paradigms. Eur J Neurosci, 35(11), 1799-1809. https://doi.org/10.1111/j.1460-9568.2012.08113.x

Schoenbaum, G., Chiba, A. A., \& Gallagher, M. (1998a). Orbitofrontal cortex and basolateral amygdala encode expected outcomes during learning. Nat Neurosci, 1(2), 155-159. https://doi.org/10.1038/407

Schoenbaum, G., Chiba, A. A., \& Gallagher, M. (1998b). Orbitofrontal cortex and basolateral amygdala encode expected outcomes during learning. Nat Neurosci, 1(2), 155-159. https://doi.org/10.1038/407

Schoenbaum, G., Chiba, A. A., \& Gallagher, M. (1999). Neural encoding in orbitofrontal cortex and basolateral amygdala during olfactory discrimination learning. J Neurosci, 19(5), 1876-1884.

Schoenbaum, G., Setlow, B., Saddoris, M. P., \& Gallagher, M. (2003). Encoding predicted outcome and acquired value in orbitofrontal cortex during cue sampling depends upon input from basolateral amygdala. Neuron, 39(5), 855-867. https://doi.org/S0896627303004744 [pii]

Sengupta, A., Yau, J. O. Y., Jean-Richard-Dit-Bressel, P., Liu, Y., Millan, E. Z., Power, J. M., \& McNally, G. P. (2018). Basolateral Amygdala Neurons Maintain Aversive Emotional Salience. J Neurosci, 38(12), 3001-3012. https://doi.org/10.1523/JNEUROSCl.2460-17.2017

Servonnet, A., Hernandez, G., El Hage, C., Rompré, P. P., \& Samaha, A. N. (2020). Optogenetic Activation of the Basolateral Amygdala Promotes Both Appetitive Conditioning and the Instrumental Pursuit of Reward Cues. J Neurosci, 40(8), 1732-1743. https://doi.org/10.1523/JNEUROSCI.2196-19.2020

Sharpe, M. J., \& Schoenbaum, G. (2016). Back to basics: Making predictions in the orbitofrontal-amygdala circuit. Neurobiol Learn Mem, 131, 201-206. https://doi.org/10.1016/j.nlm.2016.04.009

Sladky, R., Höflich, A., Küblböck, M., Kraus, C., Baldinger, P., Moser, E., Lanzenberger, R., \& Windischberger, C. (2015). Disrupted effective connectivity between the amygdala and orbitofrontal cortex in social anxiety disorder during emotion discrimination revealed by dynamic causal modeling for FMRI. Cereb Cortex, 25(4), 895-903. https://doi.org/10.1093/cercor/bht279

Stalnaker, T. A., Franz, T. M., Singh, T., \& Schoenbaum, G. (2007). Basolateral amygdala lesions abolish orbitofrontal-dependent reversal impairments. Neuron, 54(1), 51-58. https://doi.org/S0896-6273(07)00113-4 [pii] 10.1016/j.neuron.2007.02.014

Stalnaker, T. A., Liu, T. L., Takahashi, Y. K., \& Schoenbaum, G. (2018). Orbitofrontal neurons signal reward predictions, not reward prediction errors. Neurobiol Learn Mem, 153(Pt B), 137-143. https://doi.org/10.1016/j.nlm.2018.01.013

Stolyarova, A., Rakhshan, M., Hart, E. E., O'Dell, T. J., Peters, M. A. K., Lau, H., Soltani, A., \& Izquierdo, A. (2019). Contributions of anterior cingulate cortex and basolateral amygdala to decision confidence and learning under uncertainty. Nat Commun, 10(1), 4704. https://doi.org/10.1038/s41467-019-12725-1

Sugase-Miyamoto, Y., \& Richmond, B. J. (2005). Neuronal signals in the monkey basolateral amygdala during reward schedules. J Neurosci, 25(48), 11071-11083. https://doi.org/10.1523/JNEUROSCI.1796-05.2005

Suzuki, S., Cross, L., \& O'Doherty, J. P. (2017). Elucidating the underlying components of food valuation in the human orbitofrontal cortex. Nat Neurosci, 20(12), 1780-1786. https://doi.org/10.1038/s41593-017-0008-x

Takahashi, Y. K., Chang, C. Y., Lucantonio, F., Haney, R. Z., Berg, B. A., Yau, H. J., Bonci, A., \& Schoenbaum, G. (2013). Neural estimates of imagined outcomes in the orbitofrontal cortex drive behavior and learning. Neuron, 80(2), 507-518. https://doi.org/10.1016/j.neuron.2013.08.008

Tye, K. M. (2018). Neural Circuit Motifs in Valence Processing. Neuron, 100(2), 436-452. https://doi.org/10.1016/j.neuron.2018.10.001 
bioRxiv preprint doi: https://doi.org/10.1101/2021.03.20.436233; this version posted June 9, 2021. The copyright holder for this preprint (which was not certified by peer review) is the author/funder, who has granted bioRxiv a license to display the preprint in perpetuity. It is made available under aCC-BY-NC-ND 4.0 International license.

Sias et al. 31

Tye, K. M., \& Janak, P. H. (2007). Amygdala neurons differentially encode motivation and reinforcement. J Neurosci, 27(15), $3937-3945$. https://doi.org/10.1523/JNEUROSCI.5281-06.2007

Tye, K. M., Prakash, R., Kim, S. Y., Fenno, L. E., Grosenick, L., Zarabi, H., Thompson, K. R., Gradinaru, V., Ramakrishnan, C., \& Deisseroth, K. (2011). Amygdala circuitry mediating reversible and bidirectional control of anxiety. Nature, 471(7338), 358-362. https://doi.org/nature09820 [pii]10.1038/nature09820

Tye, K. M., Stuber, G. D., de Ridder, B., Bonci, A., \& Janak, P. H. (2008). Rapid strengthening of thalamo-amygdala synapses mediates cue-reward learning. Nature, 453(7199), 1253-1257. https://doi.org/10.1038/nature06963

van Duuren, E., Escámez, F. A., Joosten, R. N., Visser, R., Mulder, A. B., \& Pennartz, C. M. (2007). Neural coding of reward magnitude in the orbitofrontal cortex of the rat during a five-odor olfactory discrimination task. Learn Mem, 14(6), 446-456. https://doi.org/10.1101//m.546207

Wallis, J. D., \& Miller, E. K. (2003). Neuronal activity in primate dorsolateral and orbital prefrontal cortex during performance of a reward preference task. Eur J Neurosci, 18(7), 2069-2081.

Wassum, K. M., \& Izquierdo, A. (2015). The basolateral amygdala in reward learning and addiction. Neurosci Biobehav Rev, 57, 271283. https://doi.org/10.1016/j.neubiorev.2015.08.017

Wellman, L. L., Gale, K., \& Malkova, L. (2005). GABAA-mediated inhibition of basolateral amygdala blocks reward devaluation in macaques. J Neurosci, 25(18), 4577-4586.

Wilson, R. C., Takahashi, Y. K., Schoenbaum, G., \& Niv, Y. (2014). Orbitofrontal cortex as a cognitive map of task space. Neuron, 81(2), 267-279. https://doi.org/10.1016/j.neuron.2013.11.005

Zhou, J., Gardner, M. P. H., Stalnaker, T. A., Ramus, S. J., Wikenheiser, A. M., Niv, Y., \& Schoenbaum, G. (2019). Rat Orbitofrontal Ensemble Activity Contains Multiplexed but Dissociable Representations of Value and Task Structure in an Odor Sequence Task. Curr Biol, 29(6), 897-907.e893. https://doi.org/10.1016/j.cub.2019.01.048 
Sias et al. 32

\section{SUPPLEMENTAL FIGURES}

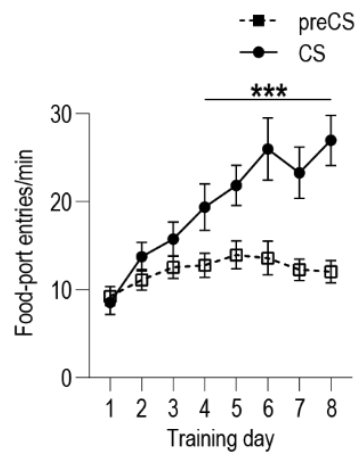

Figure 1-1. Food-port entry rate during Pavlovian conditioning for BLA fiber photometry GCaMP6f imaging experiment. Food-port entry rate (entries/min) during the CS probe period (after CS onset, before first reward delivery), averaged across trials and across the 2 CSs for each day of Pavlovian conditioning. Rats increased food-port approach responses to the CS across training (CS x Training: $F_{(7,70)}=15.31, P<0.0001$; CS: $F_{(1,10)}=48.30, P<0.0001$; Training: $\left.F_{(7,70)}=10.42, P<0.0001\right) .{ }^{* * *} P<0.0001$, relative to preCS. 
Sias et al. 33

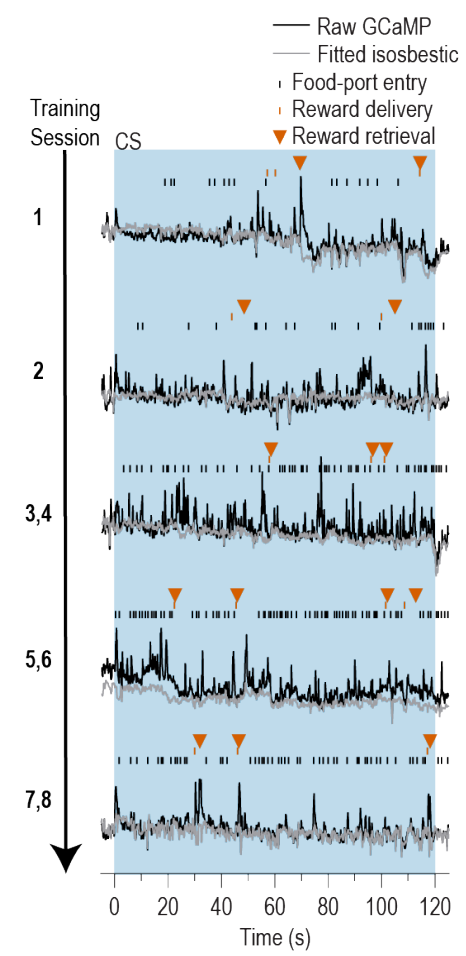

Figure 1-2. Representative examples of raw GCaMP6f and isosbestic fluorescent changes in response to cue presentation and reward delivery and retrieval across days of training. Raw GCaMP6f $(470 \mathrm{~nm}$ channel) fluorescence and corresponding fitted fluorescent trace from the isosbestic (415 nm) channel. 
Sias et al. 34

a

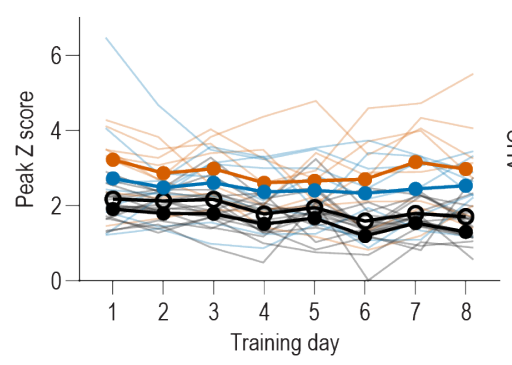

b

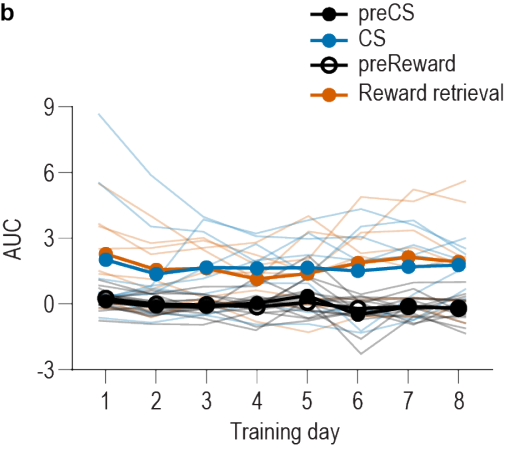

Figure 1-3. BLA neurons are activated during stimulus-outcome learning across each of the 8 Pavlovian conditioning sessions. (a-b) Trial-averaged quantification of maximal (a; peak) and area under the GCaMP Zscored $\triangle F / F$ curve (b; AUC) during the $3 \mathrm{~s}$ following $\mathrm{CS}$ onset or reward retrieval compared to equivalent baseline periods immediately prior to each event from the $N=8$ subjects for which we were able to obtain reliable recordings from each of the 8 Pavlovian conditioning sessions. Thin light lines represent individual subjects. Both $\mathrm{CS}$ and reward retrieval caused a similar elevation in the peak calcium response (Event v. baseline $F_{(0.3,1.9)}=$ 28.14, $P=0.03$; Training, Event type (CS/US), and all other interactions between factors, lowest $P=0.12)$ and area under the calcium curve (AUC; Event v. baseline $F_{(0.2,1.2)}=40.57, P=0.04$, Training, Event type (CS/US), and all other interactions between factors, lowest $P=0.21$ ) across training. Analysis of each event relative to its immediately preceding baseline period confirmed that BLA neurons were robustly activated by both the onset of the CS as reflected in the peak calcium response $\left(\mathrm{CS}: F_{(1,7)}=9.95, P=0.02\right.$; Training: $F_{(3.0,21.3)}=1.58, P=0.22$; CS x Training: $\left.F_{(1.5,10.7)}=0.43, P=0.61\right)$ and AUC $\left(C S: F_{(1,7)}=9.01, P=0.02\right.$; Training: $F_{(2.3,16.0)}=0.56, P=0.60$; CS $x$ Training: $\left.F_{(1.5,10.2)}=0.30, P=0.68\right)$, as well as at reward retrieval during the CS [(Peak, Reward: $F_{(1,7)}=$ 12.22, $P=0.01$; Training: $F_{(3.5,24.1)}=1.18, P=0.34$; Reward $x$ Training: $\left.F_{(2.5,17.4)}=1.75, P=0.20\right)$ AUC, Reward: $F_{(1,7)}=13.73, P=0.008$; Training: $F_{(2.4,17.1)}=1.19, P=0.34$; Reward $x$ Training: $\left.\left.F_{(3.0,21.3)}=2.46, P=0.09\right)\right]$. 


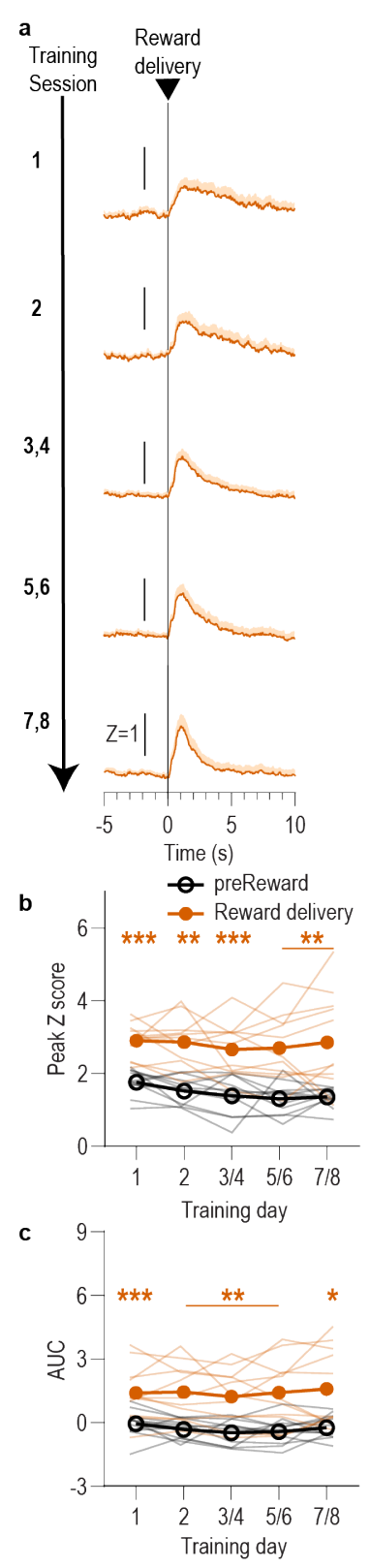

Figure 1-4. BLA reward responses aligned to reward delivery during Pavlovian conditioning. We detected a robust BLA response to reward retrieval during CS presentation during Pavlovian conditioning. This response was also detected when the data were aligned to reward delivery, which was signaled by the subtle but audible click of the pellet dispenser or sound of the sucrose pump. After initial training, reward retrieval often immediately followed reward delivery. (a) Trial-averaged GCaMP6f fluorescence (Z-scored $\Delta F / F$ ) in response to reward delivery during the CS across days of training. Shading reflects between-subjects s.e.m. Data from the last six training sessions were averaged across 2-session bins (3/4,5/6, and 7/8). (b) Trial-averaged quantification of maximal (peak) GCaMP Z-scored $\Delta F / F$ during the 3-s period following reward delivery compared to the equivalent baseline period $3 \mathrm{~s}$ prior to reward delivery. Thin light lines represent individual subjects. (c) Trialaveraged quantification of area under the GCaMP Z-scored $\triangle F / F$ curve (AUC) during the $3 \mathrm{~s}$ period following reward delivery compared to the equivalent baseline period. Across training, reward delivery caused a robust elevation in the peak calcium response (Reward delivery: $F_{(1,10)}=57.73, P<0.0001$; Training: $F_{(2.5,24.8)}=$ 1.29, $P=0.30$; Reward delivery $x$ Training: $\left.F_{(1.8,18.1)}=0.43, P=0.64\right)$ and area under the calcium curve (Reward delivery: $F_{(1,10)}=36.44, P=0.0001$; Training: $F_{(2.0,19.7)}=0.51, P=0.60$; Reward delivery $\times$ Training: $F_{(1.8,17.7)}=$ $0.39, P=0.66) . \mathrm{N}=11 .{ }^{*} P<0.05,{ }^{* *} P<0.01,{ }^{* * *} P<0.001$ relative to pre-event baseline. 


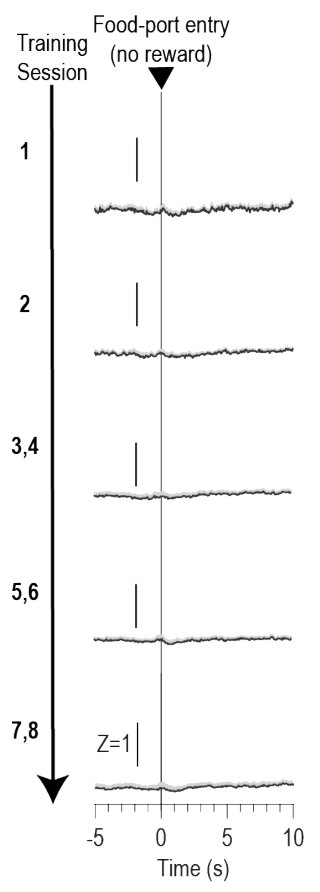

Figure 1-5. Food-port entries during the CS in the absence of reward do not trigger a BLA response. We detected a robust elevation in BLA calcium activity in response to reward retrieval during CS presentation during Pavlovian conditioning. To determine the extent to which the action of entering the food-delivery port influenced this response, we examined BLA calcium activity in response to food-port entries during the CS probe period (after CS onset, before first reward delivery). Trial-averaged GCaMP6f fluorescence (Z-scored $\Delta F / F$ ) in response to food-port entries during the CS across days of training. Shading reflects between-subjects s.e.m. Data plotted on the same scale as Figure 1 and 1-4 to facilitate comparison. Data from the last six training sessions were averaged across 2 -session bins $(3 / 4,5 / 6$, and $7 / 8) . N=11$. 

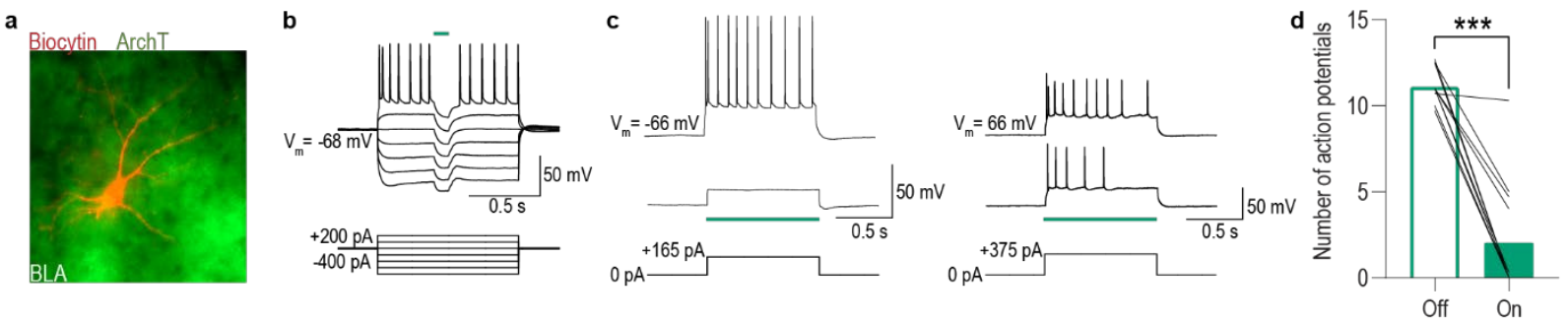

Figure 3-1. Green light activation of ArchT hyperpolarizes and attenuates the firing of BLA cells. (a) Confocal image of biocytin-filled BLA cell (red) expressing ArchT-eYFP. (b) Current-clamp recording of an ArchTexpressing BLA cell responding to hyperpolarizing and depolarizing current injections. When illuminated with green light ( $535 \mathrm{~nm}, 100 \mathrm{~ms}$ pulse, $0.5 \mathrm{~mW})$, activation of ArchT hyperpolarizes the cell membrane resulting in the absence of action potential firing at suprathreshold membrane potentials. This hyperpolarization of the cell membrane occurs only during green light luminescence. (c) Representative recordings from 2 ArchT-expressing BLA cells when injected with a suprathreshold pulse of current (165 or $375 \mathrm{pA} 1 \mathrm{~s}$; bottom) with green light off (top) or on (middle). (d) Summary of the number of action potentials recorded in ArchT-expressing BLA cells ( $N$ $=12$ cells $/ 5$ subjects) injected with a suprathreshold amount of current before (Off) and during (On) green light illumination (median $=1 \mathrm{~mW}$, range $=0.25-1$ ). Current injection intensities that resulted in 8-15 action potentials were selected for recordings (median $=275 \mathrm{pA}$, range 100-800 pA, duration $=1 \mathrm{~s}$ ). Number of action potentials was averaged across 3 sweeps/condition. Green light activation of ArchT in BLA cells reduced action potential firing in all cells and abolished ( $>97 \%$ reduction) it in most cells. The average number of action potentials recorded during green light exposure was significantly lower than the control no-light period $\left(t_{11}=9.25, P<\right.$ $0.0001)$. Lines represent individual cells. ${ }^{* * *} P<0.001$. 
Sias et al. 38
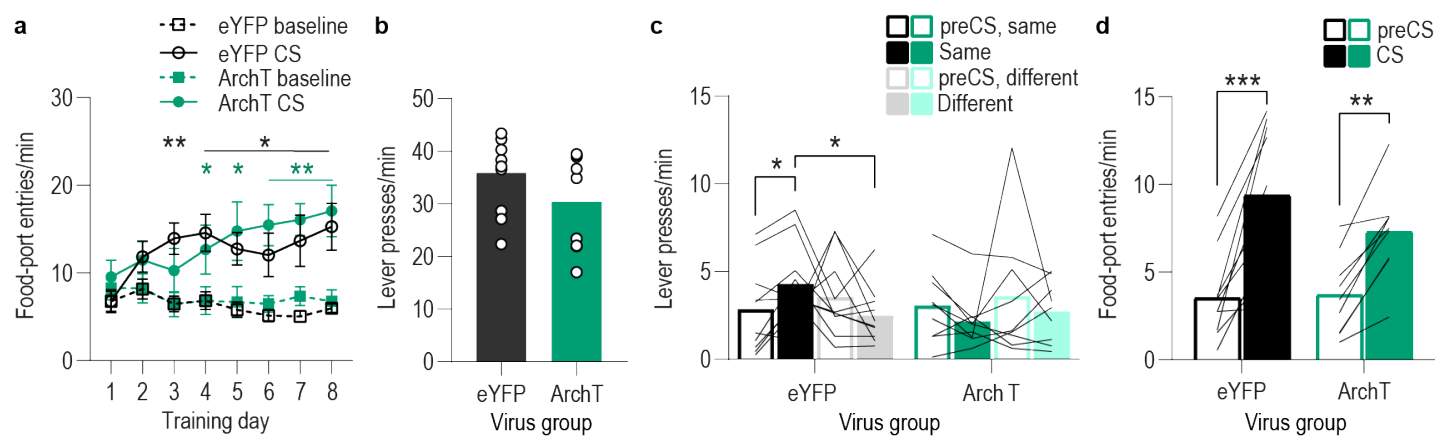

Figure 3-2. Food-port entry and press rates during Pavlovian conditioning and PIT test for BLA optical inhibition experiment. (a) Food-port entry rate (entries/min) during CS probe period (after CS onset, before first reward delivery), averaged across trials and across CSs for each day of Pavlovian conditioning. There was no effect of BLA inhibition during reward retrieval on the development of this Pavlovian conditional goal-approach response (CS x Training: $F_{(3.4,57.8)}=16.44, P<0.0001$; CS: $F_{(1,17)}=46.73, P<0.0001$; Virus: $F_{(1,17)}=0.17, P=$ 0.68; Training: $F_{(2.3,38.5)}=2.37, P=0.10$; Virus $x$ Training: $F_{(7,119)}=1.55, P=0.16$; Virus $\times$ CS: $F_{(1,17)}=0.0009, P=$ 0.98; Virus $x$ Training $\times$ CS: $\left.F_{(7,119)}=1.63, P=0.13\right)$. ${ }^{*} P<0.05,{ }^{* *} P<0.01$ relative to pre-CS. (b) Lever press rate (presses/min) averaged across levers and across the final 2 days of instrumental conditioning. There was no significant difference in press rate between the control group and the group that received BLA inhibition during Pavlovian conditioning $\left(\mathrm{t}_{17}=1.44, P=0.17\right)$. Circles represent individual subjects. (c). Lever press rate (presses/min) on the lever earning the same outcome as the presented CS (averaged across trials and CSs), relative to the press rate on the alternate lever (Different) during the PIT test. Planned comparisons (Levin et al., 1994), based on the significant interaction and post hoc effect detected in Figure 3f, showed that for the eYFP control group CS presentation significantly increased responding on the lever that earned the same reward as that predicted by the presented CS relative to the preCS baseline period $\left(t_{9}=3.11, P=0.01\right)$. The CSs did not significantly alter responses on the different lever in the control group $\left(t_{9}=1.35, P=0.21\right)$. For the ArchT group, the CSs were not capable of significantly altering lever pressing relative to the baseline period (Same: $t_{8}=2.13, P=0.07$; Different: $t_{8}=0.77, P=0.46$ ). Lines represent individual subjects. (d) Food-port entry rate during CS presentation (averaged across trials and CSs) during the PIT test. For both groups CS presentation triggered a similar elevation in this goal-approach behavior (CS: $F_{(1,17)}=59.41, P<0.0001$; Virus: $F_{(1,17)}=$ 0.63, $P=0.44$; Virus $x$ CS: $\left.F_{(1,17)}=3.42, P=0.08\right)$. Lines represent individual subjects. ${ }^{*} P<0.05,{ }^{* \star *} P<0.001$. 
Sias et al. 39

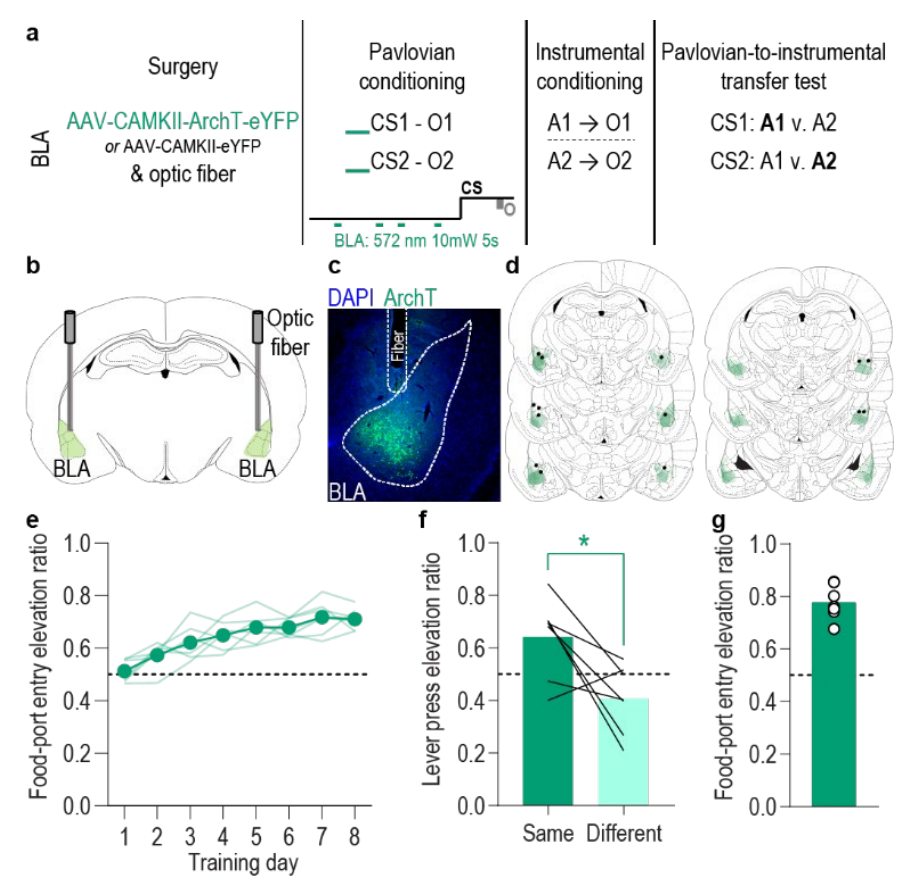

Figure 3-3. Inhibition of BLA neurons unpaired with reward delivery does not disrupt the encoding of stimulus-outcome memories. We found that inhibition of BLA neurons specifically at the time of outcome experience during each CS during Pavlovian conditioning attenuated subjects' encoding of the sensory-specific stimulus-outcome memories, as evidenced by their inability to later use those memories to guide choice behavior during a PIT test. To control for the total amount of BLA inhibition during Pavlovian conditioning, we repeated the BLA inhibition experiment in a separate group of subjects matching the frequency and duration of inhibition to the experimental group (Figure 3), but delivering it during the baseline, 2-min pre-CS periods. We selected this period for control inhibition to maintain proximity to the CS period but avoid inhibition during the CS at periods in which the rat might be expecting, checking for, and/or retrieving reward, events that were not possible for us to time. (a) Procedure schematic. CS, conditional stimulus; O, outcome (sucrose solution or food pellet); A, action (left or right lever press). (b) Schematic of optogenetic strategy for inhibition of BLA neurons. (c) Representative fluorescent image of ArchT-eYFP expression and fiber placement in the BLA. (d) Schematic representation of ArchT-eYFP expression and placement of optical fiber tips in BLA for all subjects. (e) Elevation [(CS probe entry rate)/(CS probe entry rate + preCS entry rate)] in food-port entries during CS probe period (after CS onset, before first reward delivery), averaged across trials and CSs for each day of Pavlovian conditioning. Optical inhibition of BLA neurons unpaired with reward delivery did not affect development of the Pavlovian conditional goalapproach response (Training: $F_{(3.4,20.6)}=16.83, P<0.0001$ ). Thin light lines represent individual subjects. (f) Elevation in lever presses on the lever that earned the same outcome as the presented CS (Same; [(presses on Same lever during CS)/(presses on Same lever during CS + Same presses during preCS)], averaged across trials and across CSs), relative to the elevation in responding on the alternate lever (Different; [(presses on Different lever during CS)/(presses on Different lever during CS + Different presses during preCS)], averaged across trials and CSs) during the PIT test. Inhibition of BLA neurons unpaired with reward delivery during the Pavlovian conditioning sessions did not affect the subsequent ability of the CSs to bias instrumental choice behavior during the PIT test $\left(t_{6}=2.88, P=0.03\right)$. Lines represent individual subjects. $(g)$ Elevation in food-port entries to CS presentation (averaged across trials and CSs) during the PIT test. The CSs were also capable of elevating food-port entries above baseline during the PIT test. Circles represent individual subjects. $N=7$. ${ }^{*} P<$ 0.05 , corrected post hoc comparison. 

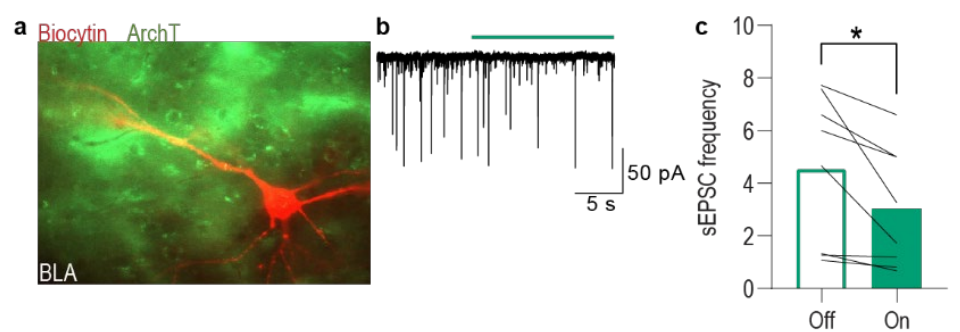

Figure 4-1. Green light activation of ArchT-expressing IOFC terminals reduces spontaneous activity in BLA neurons. (a) Confocal image of biocytin-filled BLA neuron (red) in the vicinity of ArchT-eYFP-expressing IOFC axons and terminals. (b) Representative recording of spontaneous excitatory postsynatic currents (sEPSCs) in a BLA neuron before and during green light $(535 \mathrm{~nm}, 0.5 \mathrm{~mW}, 15 \mathrm{~s}$; green bar) activation of ArchT in IOFC axonal processes. (c) Average change in sEPSC frequency in BLA cells induced by green light activation of ArchT-expressing IOFC axons and terminals in the BLA for the subset ( $N=8$ cells/ 4 subjects) of total cells ( $N$ $=12$ ) that displayed a reduction in SEPSC frequency during light. Of the remaining 4 cells, 2 showed no change in sEPSC frequency during light and 2 show an increase in frequency. Optical inhibition of IOFC terminals in the BLA resulted in a reduction in the spontaneous activity of these BLA cells $\left(t_{7}=2.92, P=0.02\right)$. Lines represent individual cells. ${ }^{*} P<0.05$. 

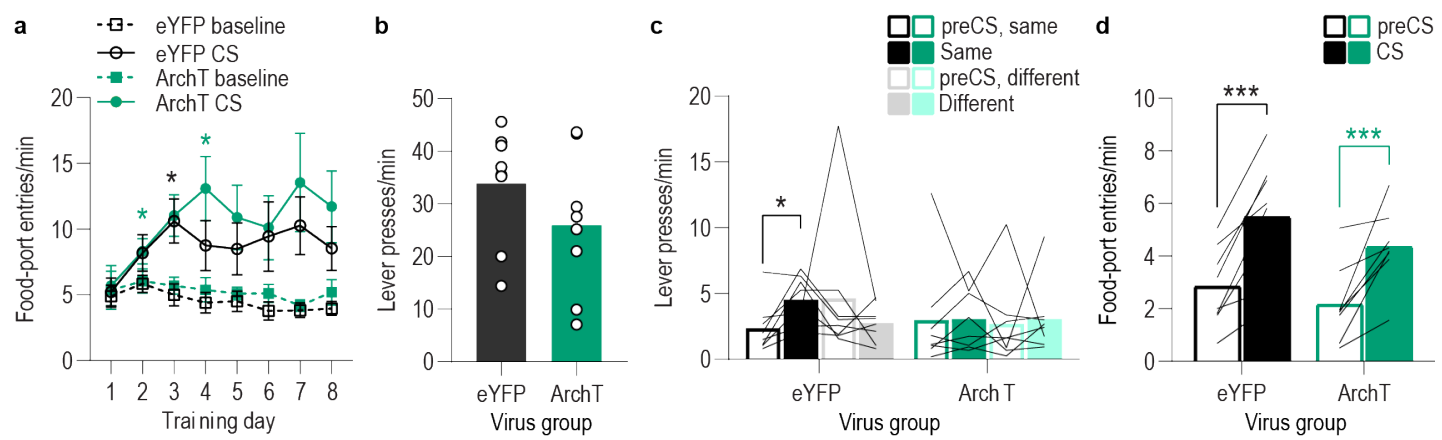

Figure 4-2. Food-port entry and press rates during Pavlovian conditioning and PIT test for IOFC $\rightarrow$ BLA optical inhibition experiment. (a) Food-port entry rate (entries/min) during CS probe period (after CS onset, before first reward delivery), averaged across trials and CSs for each day of Pavlovian conditioning. There was no effect of inhibition of IOFC $\rightarrow$ BLA projection activity during reward delivery on the development of this Pavlovian conditional goal-approach response (CS x Training: $F_{(3.5,49.1)}=5.50, P=0.002$; CS: $F_{(1,14)}=27.94, P=$ 0.0001; Virus: $F_{(1,14)}=0.82, P=0.38$; Training: $F_{(2.0,28.3)}=1.88, P=0.17$; Virus $x$ Training: $F_{(7,98)}=0.48, P=0.85$; Virus x CS: $F_{(1,14)}=0.40, P=0.54$; Virus $x$ Training $\times$ CS: $\left.F_{(7,98)}=0.62, P=0.74\right) .{ }^{*} P<0.05$, ${ }^{* *} P<0.01$ relative to pre-CS. (b) Lever press rate (presses/min) averaged across levers and across the final 2 days of instrumental conditioning. There was no significant difference in press rate between the control group and the group that received inhibition of IOFC $\rightarrow$ BLA projection activity during Pavlovian conditioning $\left(t_{14}=1.29, P=0.22\right)$. Circles represent individual subjects. (c). Lever press rate (presses/min) on the lever that earned the same outcome as the presented CS (averaged across trials and across CSs), relative to the press rate on the alternate lever (Different) during the PIT test. Planned comparisons, based on the significant interaction and post hoc effect detected in Figure 4f, showed that for the eYFP group CS presentation significantly increased responding on the lever that earned the same reward as that predicted by the presented CS relative to the preCS baseline period $\left(t_{7}=3.16, P=0.02\right)$. The CSs did not significantly alter responses on the different lever in the control group $\left(t_{7}=\right.$ $1.05, P=0.33)$. For the ArchT group, the CSs were not capable of significantly altering lever pressing relative to the baseline period (Same: $t_{7}=0.07, P=0.95$; Different: $t_{7}=0.22, P=0.83$ ). Lines represent individual subjects. (d) Food-port entry rate during CS presentation (averaged across trials and across CSs) during the PIT test. For both groups CS presentation triggered a similar significant elevation in this goal-approach behavior $\left(C S: F_{(1,14)}=\right.$ 49.96, $P<0.0001$; Virus: $F_{(1,14)}=1.35, P=0.26$; Virus x CS: $\left.F_{(1,14)}=0.44, P=0.52\right)$. Lines represent individual subjects. ${ }^{*} P<0.05,{ }^{* * *} P<0.001$. 
Sias et al. 42

a

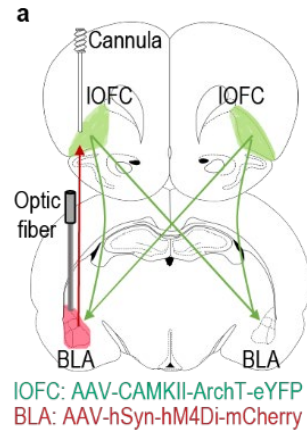

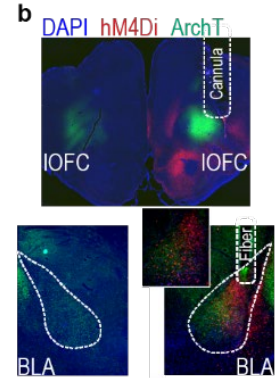

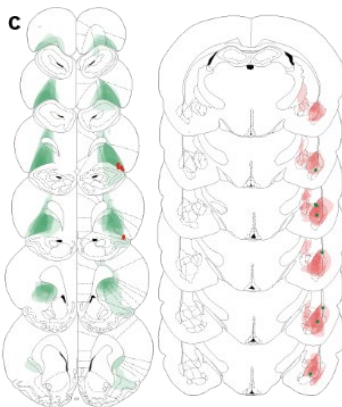

Figure 5-1. Histological verification for unilateral, ipsilateral IOFC $\rightarrow B L A / B L A \rightarrow I O F C$ inhibition subjects. (a) Schematic of multiplexed optogenetic/chemogenetic inhibition strategy for unilateral optical inhibition of IOFC $\rightarrow$ BLA projections during Pavlovian conditioning and ipsilateral, unilateral, chemogenetic inhibition of $\mathrm{BLA} \rightarrow$ IOFC projections during the PIT test. (b) Top: Representative fluorescent image of ArchT-eYFP expression in IOFC cells bodies and unilateral expression of hM4Di-mCherry in BLA axons and terminals in the IOFC in the vicinity of implanted guide cannula. Bottom: Representative image of fiber placements in the vicinity of immunofluorescent ArchT-eYFP expression in IOFC axons and terminals in the BLA and unilateral expression of hM4Di-mCherry in BLA cell bodies in that same hemisphere. (c) Schematic representation of bilateral ArchTeYFP expression and unilateral cannula placement in IOFC and unilateral, ipsilateral hM4Di expression and placement of optical fiber tips in BLA for all subjects. All fibers and cannula are shown in left hemisphere, but inhibited hemisphere was counterbalanced across subjects. 

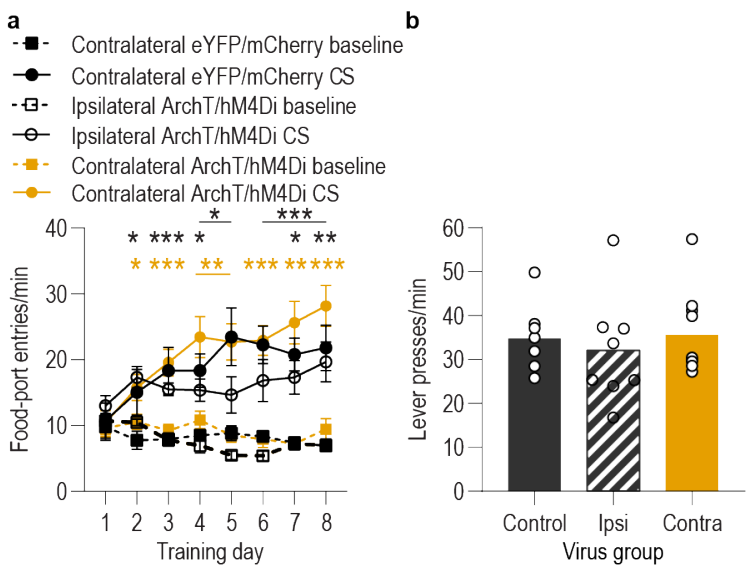

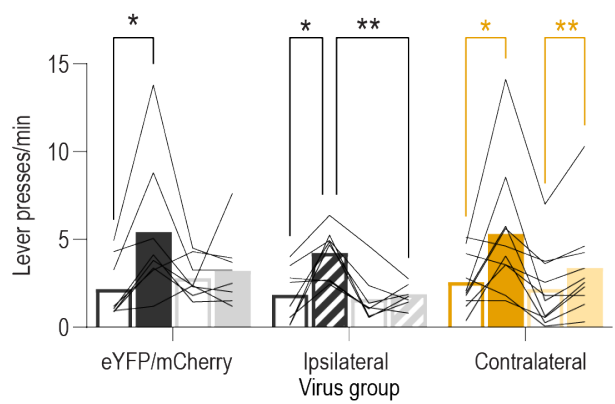

d
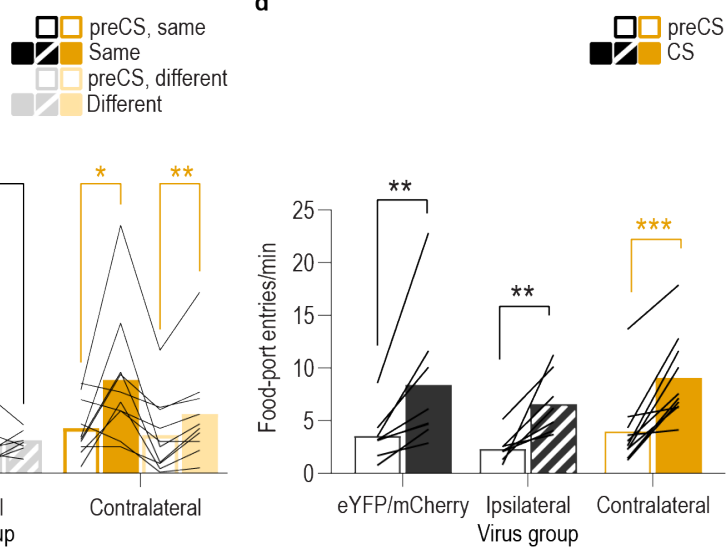

Figure 5-2. Food-port entry and press rates during Pavlovian conditioning and PIT test for IOFC $\rightarrow$ BLA/BLA $\rightarrow$ IOFC serial disconnection experiment. (a) Food-port entry rate (entries/min) during CS probe period (after CS onset, before first reward delivery), averaged across trials and CSs for each day of Pavlovian conditioning. There was no effect of unilateral IOFC $\rightarrow B L A$ inhibition during reward delivery on the development of this Pavlovian conditional goal-approach response in either the disconnection or ipsilateral control group (CS x Training: $F_{(3.4,78.6)}=23.07, P<0.0001$; CS: $F_{(1,23)}=131.7, P<0.0001$; Virus group: $F_{(2,23)}=$ 1.42, $P=0.26$; Training: $F_{(3.7,85.4)}=3.95, P=0.007$; Virus $x$ Training: $F_{(7.4,85.43)}=2.24, P=0.04$; Virus $\times$ CS: $F_{(2,23)}=1.19, P=0.32$; Virus $x$ Training $x$ CS: $\left.F_{(6.8,78.6)}=1.36, P=0.24\right) .{ }^{* *}<0.05,{ }^{* *} P<0.01,{ }^{* * *} P<0.001$, relative to preCS (top, eYFP/mCherry; middle, ipsilateral ArchT/hM4Di; bottom, contralateral ArchT/hM4Di). (b) Lever press rate (presses/min) averaged across levers and across the final 2 days of instrumental conditioning. There was no significant difference in press rate between the control groups and the disconnection group $\left(F_{(2,23)}\right.$ $=0.30, P=0.75$ ). Circles represent individual subjects. (c). Lever press rate (presses $/ \mathrm{min}$ ) on the lever that earned the same outcome as the presented CS (averaged across trials and across CSs), relative to the press rate on the alternate lever (Different) during the PIT test. Planned comparisons, based on the results detected in Figure 5f, showed that for the contralateral eYFP/mCherry control subjects CS presentation significantly increased responding on the action earning the same reward as that predicted by the presented cue relative to the preCS baseline period $\left(t_{7}=3.30, P=0.01\right)$. The CSs did not significantly alter responses on the different lever in this group $\left(t_{7}=0.58, P=0.58\right)$. For the ipsilateral ArchT/hM4Di control subjects, CS presentation increased responding on the Same action relative to both the preCS baseline period $\left(t_{7}=3.43, P=0.01\right)$ and to the different action during the CS $\left(t_{7}=4.51, P=0.003\right)$. The CSs also did not significantly alter responses on the different lever in this control group $\left(t_{7}=0.67, P=0.52\right)$. For the Disconnection (contralateral ArchT/hM4Di) group, the CSs caused a non-discriminate increase in lever pressing relative to the baseline period on both levers (Same: $t_{9}=2.54, P=0.03$; Different: $t_{9}=3.92, P=0.004$ ). Lines represent individual subjects. (d) Food-port entry rate during CS presentation (averaged across trials and across CSs) during the PIT test. For all groups, CS presentation triggered a similar significant elevation in this goal-approach behavior $\left(\mathrm{CS}: F_{(1,23)}=47.67, P<\right.$ 0.0001; Virus: $F_{(2,23)}=0.86, P=0.44$; Virus x CS: $\left.F_{(2,23)}=0.14, P=0.87\right)$. Lines represent individual subjects. Contra, contralateral. ${ }^{*} P<0.05,{ }^{* *} P<0.01,{ }^{* * *} P<0.001$. 